C2017, Elsevier. Licensed under the Creative Commons Attribution-NonCommercialNoDerivatives 4.0 International http://creativecommons.org/about/downloads

(c) $\oplus \Theta \Theta$ 


\title{
D2/D3 dopamine receptor binding with [F-18]fallypride correlates of executive function in medication-naïve patients with schizophrenia
}

\author{
Nora S Vyas ${ }^{\mathrm{a}, \mathrm{b}^{*}}$, Monte S Buchsbaum ${ }^{\mathrm{c}}$, Douglas S Lehrer ${ }^{\mathrm{d}}$, Brian M Merrill ${ }^{\mathrm{d}}$, Alex DeCastro ${ }^{\mathrm{c}}$, \\ Nicholas A Doninger ${ }^{\mathrm{e}}$, Bradley T Christian ${ }^{\mathrm{f}}$, Jogeshwar Mukherjee ${ }^{\mathrm{g}}$
}

${ }^{a}$ Kingston University London, Department of Psychology, Penrhyn Road, Kingston-uponThames, Surrey, KT1 2EE, UK.

${ }^{\mathrm{b}}$ Department of Nuclear Medicine, Charing Cross Hospital, Imperial College Healthcare NHS Trust, Fulham Palace Road, W6 8RF, UK.

${ }^{\mathrm{c}}$ University of California, San Diego, Departments of Psychiatry and Radiology, 11388 Sorrento Valley Road, San Diego, 92121, California, USA.

${ }^{\mathrm{d}}$ Wright State University Boonshoft School of Medicine, Department of Psychiatry, East Medical Plaza, 627 South Edwin C. Moses Blvd., Dayton, OH 45408, USA.

${ }^{\mathrm{e}}$ Wright State University Boonshoft School of Medicine, Department of Psychiatry, WallaceKettering Neuroscience Institute, 3533 Southern Blvd, Kettering, Ohio, 45429, OH, USA.

${ }^{\mathrm{f}}$ University of Wisconsin-Madison, Waisman Laboratory for Brain Imaging, 1500 Highland Avenue, Madison, WI, 53705, USA.

${ }^{\mathrm{g}}$ University of California, Irvine, Preclinical Imaging, Department of Radiological Sciences, California, 92697-5000.

*Corresponding author: Dr Nora S Vyas, Kingston University London, Department of

Psychology, Penrhyn Road, Kingston-upon-Thames, Surrey, KT1 2EE, UK. Tel: +44 (0) 208

417 2339; Email: n.vyas@kingston.ac.uk 


\begin{abstract}
Converging evidence indicates that the prefrontal cortex is critically involved in executive control and that executive dysfunction is implicated in schizophrenia. Reduced dopamine D2/D3 receptor binding potential has been reported in schizophrenia, and the correlations with neuropsychological test scores have been positive and negative for different tasks. The aim of this study was to examine the relation between dopamine D2/D3 receptor levels with frontal and temporal neurocognitive performance in schizophrenia. Resting-state ${ }^{18} \mathrm{~F}$-fallypride positron emission tomography was performed on 20 medication-naïve and 5 previously medicated for brief earlier periods patients with schizophrenia and 19 age- and sex-matched normal controls. Striatal and extra-striatal dopamine D2/D3 receptor levels were quantified as binding potential using fallypride imaging. Magnetic resonance images in standard Talairach position and segmented into gray and white matter were co-registered to the fallypride images, and the AFNI stereotaxic atlas was applied. Two neuropsychological tasks known to activate frontal and temporal lobe function were chosen, specifically the Wisconsin Card Sorting Test (WCST) and the California Verbal Learning Test (CVLT). Images of the correlation coefficient between fallypride binding and WCST and CVLT performance showed a negative correlation in contrast to positive correlations in healthy volunteers. The results of this study demonstrate that lower fallypride binding potential in patients with schizophrenia may be associated with better performance. Our findings are consistent with previous studies that failed to find cognitive improvements with typical dopamine-blocking medications.
\end{abstract}

Keywords: Cognition; cortex; memory; positron emission tomography; prefrontal cortex. 


\section{Introduction}

Neurocognitive impairments are a core feature of schizophrenia, with the largest effect sizes (greater than $1.5 \mathrm{z}$-score differences) reported in both executive function and verbal memory (Bilder et al., 2000; Dickinson and Harvey, 2009; Reichenberg et al., 2010; Vyas et al., 2012). Impairments in executive function are found to predict poor functional outcome, failure in treatment interventions and limited rehabilitation (Green et al., 2000). Executive function includes multiple cognitive constructs regulated by the coordination of multi-operational neural systems and is a linchpin of cognitive ability (Bressler and Menon, 2010). It has been linked in functional neuroimaging studies to the fronto-striatal-thalamic network thought to be associated with the pervasive cognitive deficits reported in schizophrenia (Andreasen, 1997; Andreasen et al., 1997; Buchsbaum et al., 1999; Hazlett et al., 2008; Kessler et al., 1993). This network, with its detailed neuroanatomical structure (Alexander et al., 1986) is critical for performing tasks such as the Wisconsin Card Sorting Test (WCST) that are known to elicit performance impairments in schizophrenia, and its dysfunctional operation demonstrated in unmedicated schizophrenia patients confirmed with functional brain imaging approaches (see review and meta-analysis (Buchsbaum et al., 2005). Abnormalities in the fronto-striatal-thalamic network have also been demonstrated in functional magnetic resonance imaging (fMRI) studies of psychosis-prone individuals (Ettinger et al., 2013; Kumari et al., 2008).

While the striatum and thalamus are known to have the highest dopamine receptor concentrations in the brain, the frontal and temporal lobes also contains significant numbers of receptors on rigorous postmortem examination (Joyce et al., 1991). High-affinity dopamine receptor ligands developed for positron emission tomography (PET) make it possible to assess the fronto-striato-thalamic distribution of dopamine binding. In healthy subjects significant 
extra-striatal binding has been demonstrated (Buchsbaum et al., 2006; Farde et al., 1997; Lehrer et al., 2005; Mukherjee et al., 2002). In unmedicated and never-medicated subjects with schizophrenia, abnormalities in extra-striatal dopaminergic neurotransmission have been reported in the prefrontal cortex (Lehrer et al., 2010a) and the medial dorsal nucleus of the thalamus (Buchsbaum et al., 2006; Kessler et al., 2009; Lehrer et al., 2005; Suhara et al., 2002; Talvik et al., 2003; Yasuno et al., 2004). The earliest report by Suhara and colleagues (Suhara et al., 2002) showed lower binding potential in the thalamus in patients with schizophrenia compared with controls (in retrospect, a one-tailed test would have been $p=0.03$; see their Table 1). A single photon emission computed tomography (SPECT) study using epidepride (Glenthoj et al., 2006) failed to detect binding reduction in the thalamus in patients with schizophrenia, however this negative finding may be due to lower resolution in SPECT imaging. In our studies with higher-resolution PET the decreased binding in schizophrenia was most marked in the medial dorsal nucleus isolated by tracing on co-registered MRI (Lehrer et al., 2010b), whereas in healthy subjects, the medial dorsal nucleus showed relatively higher binding compared with other thalamic nuclei (Takahashi et al., 2006).

There is increasing evidence that patients with schizophrenia exhibit impairment on executive function tasks, such as the WCST and the California Verbal Learning Test (CVLT), which is precisely aligned to non-motor fronto-striatal-thalamic circuits. Performance deficits in WCST categories achieved and perseverative errors has been widely documented in firstepisode psychosis (Addington et al., 2003; Bilder et al., 2000; Brickman et al., 2004; Fagerlund et al., 2006; Mohamed et al., 1999; Riley et al., 2000; Townsend et al., 2001), and metaanalyses (Fatouros-Bergman et al., 2014; Fioravanti et al., 2005; Li, 2004). Functional MRI and PET frontal activation deficits have been found in schizophrenia (Berman et al., 1995; Patel et 
al., 2010; Riehemann et al., 2001) and an activation likelihood estimation meta-analysis on a series of fMRI studies using the WCST identified the frontal lobe, thalamus and striatum as important activation sites (Buchsbaum et al., 2005). The prevailing evidence from imaging studies suggests more widespread brain activations during WCST performance (Barcelo et al., 1997; Nyhus and Barcelo, 2009). An EEG study of healthy individuals revealed increased activity during task performance across all frequency bands in the frontal and temporo-parietooccipital regions (except alpha), indicating that executive functioning skills underlying performance on the WCST may represent the operation of a more complex and widespread communication network (Gonzalez-Hernandez et al., 2002). Since dopamine projections are widespread, dopamine deficits may have profound effects on higher-order cognition (Vyas et al., 2011).

The CVLT, a measure of verbal learning and memory that relies on serial-order and semantic-cluster strategies, also involves frontal areas, as demonstrated in patients with focal lesions (Alexander et al., 2003; Baldo et al., 2002), striatal activity in PET studies of normal aging (Brickman et al., 2003), and reduced functional connectivity between the medial prefrontal, thalamus and striatum in fMRI studies of mild cognitive impairment (Liang et al., 2011). Deficits in the CVLT have consistently been reported in early-onset (Brickman et al., in press; McClellan et al., 2004; Oie and Rund, 1999; Roofeh et al., 2006), first-episode (Bilder et al., 2000; Hill et al., 2001; Saykin et al., 1994), chronic / multiple-episode (Aleman et al., 1999; Heinrichs and Zakzanis, 1998) patients with schizophrenia, high risk studies (Hill et al., 2004; Paulsen et al., 1995), and a meta-analysis (Szoke et al., 2008).

Four resting [F-18]fallypride PET studies in schizophrenia have reported low dopamine receptor binding potential in the medial dorsal nucleus of the thalamus (Buchsbaum et al., 2006; 
Kessler et al., 2009; Lehrer et al., 2010a; Yasuno et al., 2004), and in the prefrontal cortex in patients with schizophrenia (Lehrer et al., 2010a). A study using the high-affinity radioligand [11C]FLB 457 PET also showed low D2/D3 receptor binding in the right medial thalamus in drug-naïve patients with schizophrenia compared with controls (Talvik et al., 2003).

Since excess dopaminergic activity has been hypothesized to be involved in the pathophysiology of schizophrenia, we might expect that poor WCST performance would be associated with a more active dopamine system. In subjects with schizophrenia, higher ${ }^{18} \mathrm{~F}-$ FDOPA uptake has been associated with poor task performance on the WCST perseverative errors (Meyer-Lindenberg et al., 2002). Another study showed that more perseverative errors on the CANTAB Set shifting task were significantly associated with lower binding potential in patients with schizophrenia (Fagerlund et al., 2013). Ghahremani and colleagues reported better (shorter) stop-signal reaction time associated with higher fallypride binding potential in the striatum (Ghahremani et al., 2012).

Animal models have demonstrated that the striatum plays an important role in decisionmaking and performing goal-directed actions (Ito and Doya, 2009). Human choice behavior has been conceptualized as based on two decision systems, namely model-free and model-based, where the former system is influenced by making predictions based on previous experiences while the latter goal-directed system plans a set of actions based on future prospects. A recent study used a two-step decision choice task during fMRI combined with 18F-FDOPA PET in healthy participants, reported that higher levels of presynaptic dopamine in the ventral striatum was associated with greater model-based coding in the lateral prefrontal cortices (Deserno et al., 2005). Importantly, Ito et al. observed a significant negative correlation between the 
binding potential of dopamine D2 receptors and endogenous dopamine synthesis rate (Ito et al., 2011) indicating that high dopa uptake and low binding potential are alternate assessments.

The aim of the current study was to investigate the relationship between dopamine D2/D3 receptor density, as indexed by $\left[{ }^{18} \mathrm{~F}\right]$ fallypride, and executive function (as measured by the WCST), and episodic memory (as measured with CVLT), in a group of medication-naïve patients with schizophrenia and matched healthy volunteers. Because previous studies have shown low dopamine D2/D3 receptor binding potential in schizophrenia, we hypothesized that 1) there will be a positive association between high binding potential and performance in healthy volunteers 2) this association will be less pronounced in patients with schizophrenia due to dysfunctional dopamine deployment in the frontal cortex, 3) heterogeneous dopamine deficits in patients with schizophrenia will diminish regional brain fallypride binding potential vs. neuropsychological performance correlations, 4) there will be an association between binding potential and short-delay recall (model-free) in the ventral striatum in healthy volunteers, while a lower correlation will be found between binding potential and WCST categories achieved (model-based). 


\section{Material and methods}

\subsection{Subjects}

Twenty-five patients with schizophrenia (18 men; 7 women; age mean \pm SD: $31.6 \pm 12.2$ ) were recruited from the greater Dayton, Ohio area, and assessed and consented as previously described (Buchsbaum et al., 2006; Lehrer et al., 2010a). We clinically evaluated participants (Buchsbaum et al., 2006) (n=15) using the Comprehensive Assessment of Symptoms and History (Andreasen et al., 1992) and the Brief Psychiatric Rating Scale (Overall, 1972, 1976). We clinically assessed later subjects $(n=18)$ using the Positive and Negative Syndrome Scale (Kay et al., 1987), from which BPRS scores could be estimated for the purpose of combining all subjects. Over the period of subject acquisition, other additional projects required the PANNS and the BPRS was inadvertantly not obtained on a few subjects. However the neuropsychological testing was maintained in a standard way throughout this period. A boardcertified psychiatrist (D.S.L.) diagnosed all subjects using the Structured Clinical Interview for DSM-IV Axis I Disorders (First et al., 1996). A board-certified psychiatrist (author D.S.L.) diagnosed all subjects. He performed serial standard clinical psychiatric examinations on each and utilized available collateral information (family, treating clinicians, medical records). $\mathrm{He}$ confirmed each diagnosis using the Structured Clinical Interview for DSM-IV Axis I Disorders (SCID-I). We followed the very few schizophreniform subjects (i.e., duration of illness less than six months at the time of study) in order to determine whether they eventually met criteria for schizophrenia; this diagnostic conversion occurred in all such cases. Twenty-one subjects were diagnosed with schizophrenia and four with schizoaffective disorder. Twenty patients were absolutely neuroleptic naïve, as confirmed by case notes and psychiatric interview, and five were previously medicated, although only three had a lifetime neuroleptic exposure of greater than 
two weeks. No subject had received neuroleptic within 30 days of study. This was verified in most cases with both clinical records and information obtained from relatives. Subject characteristics are summarized in Table 1 . This sample size is slightly smaller than our previous report (Lehrer et al., 2010a) because in the process of reregistering the FDG-MRI-diffusiontensor-fallypride images a small number of subjects did not have the complete dataset.

Nineteen normal control subjects (12 men; 7 women; mean age \pm SD: $29.2 \pm 9.3$ ) were recruited via newspaper and poster advertisement. Controls were age- and sex-matched to experimental subjects. The exclusion criteria for the control group included: (a) personal or family history of psychiatric illness, or intellectual disabilities; (b) personal or family history of hereditary neurological disorders, previous brain infections, Parkinson's disease, significant medical disorders, or head injury resulting in a loss of consciousness for more than $1 \mathrm{~h}$, and (c) DSM-IV criteria for psychoactive substance abuse in the preceding 6 months or the presence of co-morbid Axis I disorders.

\subsection{Cognitive assessment}

\subsubsection{Executive function}

A computerized version of the WCST:CV4 (Heaton, 2003; Milner, 1963) was administered to all participants, who were instructed to match a response card to one of the four reference cards on the basis of three perceptual dimensions (shape, color, or number). Subjects were not informed of the correct sorting principle but were provided feedback on the response ('Right' or 'Wrong'). The following performance indexes were of interest: (1) perseverative errors scored as number of repetitive errors; (2) total errors: total number of perseverative and non-perseverative errors (presented as T-scores with positive values indicating better 
performance as fewer errors); (3) categories achieved: number of rules that have been correctly identified; and (4) extradimensional shift learning: loss of set or failure to maintain set. For correlation analyses, perseverative errors and shift learning signs were reversed so that all four scores had the direction of higher values with higher performance.

\subsubsection{Verbal learning and memory}

Verbal learning and memory were assessed with the CVLT (Delis et al., 1987; Delis, 2000) in which a list of 16 words (List A) is read to the participant and they are instructed to recall as many items in any order on five consecutive trials. Each item can be classified into semantic categories (spices \& herbs, tools, fruits, or clothing). Following trials 1-5, the participant is presented with an interference list of 16 new words (List B) over a single trial, immediately followed by a short-delay free recall trial. After a 20 minute delay, the participant is required to recall List A. Responses that do not match the target list during the recall trials are denoted intrusions. A 44-item yes-no recognition test for List A is assessed following the long delay recall trial. The outcome variables included total words recalled for trials $1-5$, short-delay free recall, long-delay free recall (20 minute delay), learning slope, recognition hits, false alarms, response bias, and discriminability. The CVLT also provides indexes to assess learning strategy, including semantic clustering (recalling the items consecutively in categories) and serial clustering (recalling items in the order in which they are presented). 


\subsection{Imaging acquisition}

\subsubsection{PET Scan}

The subjects refrained from alcohol and caffeine 4 hours before the PET scanning session. The PET scans were acquired using an ECAT EXACT HR+ scanner in 3D mode (Brix et al., 1997).The subjects were placed in the scanner in a supine position. The subjects' head was fixed with a surgical tape as described earlier (Lehrer et al., 2010a). A 5-minute transmission scan was first acquired using a ${ }^{68} \mathrm{Ge} /{ }^{68} \mathrm{Ga}$ rod source to correct for the attenuation of photons. The dynamic acquisition of the PET dynamic data was initiated with a 30 -second bolus injection of ${ }^{18} \mathrm{~F}$ fallypride. The radiopharmaceutical was produced according to previously reported methods (Mukherjee et al., 1995) at high specific activity (> $2800 \mathrm{Ci} / \mathrm{mmole}$ ) with a dose of $0.7 \mathrm{mCi} / 10 \mathrm{~kg}$ (range of $3.7-7.4 \mathrm{mCi}$ ). The initial subject cohort underwent two hours of dynamic PET imaging, while the later studied participants were scanned for three hours from start to finish, with a 20minute rest period mid-scan. The data were reconstructed using the ECAT v7.2 OSEM following corrections applied for attenuation, normalization, and scatter. Before further data processing, the dynamic frames were spatially aligned using AIR 3.08 software (Woods et al., 1992) was used to correct for head motion. The cerebellum was used to represent the reference region (devoid of specific ligand-receptor binding) for the data analysis. Multiple, circular regions of interest were placed over the outer lobules of the cerebellum, covering two 3.5-mm-thick transaxial planes to obtain cerebellar values.

Co-registration to anatomical images was done by using early sum images. We registered the distribution volume ratio images to the MNI template (12-parameter affine registration model using FLIRT) and examined the individual standardized images. Thirty-seven achieved good registration, so a standard fallypride template was created from these individuals. Each fallypride 
image was then reregistered to the mean fallypride template. Next the transformation matrix for fallypride template to MNI standard brain was used to transform every fallypride subject to MNI space. We obtained binding potential uptake assessments using the analysis of functional neuroimages (AFNI) regions of interest.

We examined the quality of co-registration by locating the edge of the caudate nucleus using an $x$-dimension voxel strip, and found the first derivatives of the binding potential (BP). The MRI were aligned within $1 \mathrm{~mm}$ in $82 \%$ of cases and within $2 \mathrm{~mm}$ in $16 \%$ of cases, indicating good co-registration. We further assessed the binding potential computation, coregistration to MNI template and AFNI region of interest reliability on an independent series of fallypride scans of healthy volunteers and patients with schizophrenia collected at UCSD. Each of the three steps was carried out using similar or identical scripts. Scans at UCSD were collected as four frames and the process was applied to the odd and even frame totals independently, thus allowing assessment of all three steps each with attendant error. Since the counts were only approximately half as great, this might provide an underestimate of reliability. Product-moment correlations for reliability for the right and left nucleus accumbens were 0.99, 0.99 and for right with left, 0.89 and 0.79 . The odd-frame left vs. even frame right correlations remained acceptable and are both anatomically independent (since they are more than 10 fullwidth half-maximum apart) and statistically independent (entirely separate samples). Right vs. left pairings were sensitive to misalignment in structures near the edge, since a right/left shift can place part of the structure in CSF on one side but not the other. However, right-left pairs remained highly reliable even when they were not adjacent to brain edges. Other areas were similarly reliable, including cortical areas. 


\subsubsection{MR imaging}

T1 weighted MRI images (1.5T) were acquired on a GE Signa NVi system (General Electric, Milwaukee) using the following parameters, TR=24 msec, TE $=5 \mathrm{~ms}$, flip angle $=40^{\circ}$, slice thickness $=1.2 \mathrm{~mm}$, pixel matrix $=256 \times 256$, field of view $=23 \mathrm{~cm}$, total slices $=128$.

\subsection{Data analysis}

Our strategy was to preselect AFNI anatomical areas associated with imaging abnormalities in schizophrenia and task performance. We prioritized the striatum, thalamus, and prefrontal cortex for analysis. Statistical analysis was performed using IBM Statistical Package for the Social Sciences (SPSS v.21). Chi-square and Welch $t$-tests were applied to assess differences in demographic characteristics and cognitive performance in patients with schizophrenia in comparison with matched healthy volunteers. Multiple regression was carried out with $\mathrm{R}$ and package psych and missing data were imputed with $\mathrm{R}$ package e1071 which substituted the mean value for all subjects. All brain imaging values were present and all neuropsychological values were present for healthy controls but there were but there were 4 Wisconsin Cart Sort Tests and 4 CVLT scores missing from the patient group (not both in the same patient in 2). The sample is slightly smaller than our previous report (Lehrer et al., 2010 because we chose the individuals who had four images (fallypride, MRI, FDG, and fractional anisotropy) for planned multimodal analysis.

Power was assessed by examining published dopamine BP correlations with task

performance. The median explained variance with the CANTAB battery (Fagerlund et al., 2013) was $25.7 \%$, corresponding to a correlation coefficient of 0.51 . Our power to detect a significant correlation of 0.51 in 25 patients is 0.75 (Cohen, 1969, p 99). Using CANTAB set-shifting as a 
proxy for the Wisconsin Card Sort Test, the $\mathrm{z}$ difference in correlations in patients with schizophrenia and healthy volunteers was approximately 1.00 , which corresponds to a power of .91 for healthy/patient differences (Cohen, 1969, p 121)

We calculated the distribution of correlation coefficients (scores by z-transformed ROI matrix) and compared the distributions with the Kolmogorov-Smirnov distribution and Welch ttest (ks.test, in R). The rectangular portion of the correlation matrix for neuropsychological scores vs. fallypride binding potential was compared between healthy and patient groups using a R language simulation since the regional binding potential and CVLT variables were correlated. We carried out 1000 random permutation simulations using the 44 subjects to form 1000 groups of 19 and 25 subjects, calculated the test statistic on each iteration, and used the resulting table of the 1000 test statistics to test the significance of the healthy vs. patient correlation matrix differences. Thus the differences in correlation matrices between healthy and patient groups were confirmed statistically with a single permutation probability test.

Maps of r correlations and Fisher $\mathrm{Z}$ tests to compare correlation maps are presented. A color bar from 0.05 to 0.001 is given to allow the reader to compare already published binding potential/task performance findings with ours as one-tailed confirmations or exploratory analysis depending on the viewer's goal. These maps may be considered as 1) analogous to follow-up anova in a two factor repeated measures design where follow-up t-tests on individual cells are performed to localize the greatest effects, 2) confirmatory of published binding potential vs cognitive performance correlations or 3) exploratory presentation of regional brain effects. The overall average $\mathrm{Z}$ differences were confirmed by permutation analysis as described above.

\subsubsection{Binding potential parametric image analysis}


The BP values reported here were calculated using a multilinear variation (Ichise et al., 2002) of the Logan (Logan et al., 1996) distribution volume ratio (DVR). This model employs the use of a tissue reference region (cerebellum) to represent the kinetics of unbound radioligand in the tissue (Cunningham et al., 1991; Logan et al., 1996). More details of these methods can be found in our previous report (Buchsbaum et al., 2006).

\subsubsection{Regions of interest}

Selective AFNI regions of interest (ROIs) were calculated from each subject. We calculated product-moment correlation coefficients between each ROI and the brain areas. For the neuropsychological measures we chose four WCST scores (categories achieved, perseverative errors, total correct, extradimensional set shifting) and nine scores for the CVLT. Based on earlier findings of fallypride binding reduction in the thalamus, frontal lobe and temporal lobe, on commonly reported regions showing brain imaging abnormalities, and the frontostriatal dopamine relationships shown in combined PET/diffusion tensor imaging studies (Tziortzi et al., 2014), we focused on 13 regions: the dorsal medial and lateral nuclei of the thalamus, the pulvinar, the medial and lateral geniculate, three superior/inferior levels of the caudate, the anterior (Brodmann area (BA) 32), and posterior cingulate (BA23), anterior cingulate cortex (BA24), and the medial and lateral globus pallidus. For an alternate view of dopaminergic activity, we chose the dorsolateral prefrontal region, and the medial and lateral temporal lobe, also regions widely confirmed as different in patients with schizophrenia (hippocampus, uncus, parahippocampus, lingual gyrus, and Brodmann areas: 8, 9, 10, 44, 45, 46, 47, 20, 21, 22) (Buckley, 2005; Davidson and Heinrichs, 2003; Ellison-Wright et al., 2008; Glahn et al., 2008). 



\section{Results}

\section{1. [F-18] Fallypride and WCST}

In the fronto-striatal-thalamic region set, there was no significant difference between healthy volunteers and patients on the four WCST scores and no difference was confirmed with the Hotelling $\mathrm{T} 2$ test $(F=3.08, \mathrm{df}=4,39, p=0.23)$. The Hotelling $\mathrm{T} 2$ was at a trend level for the 13 BP values $(F=1.17, \mathrm{df}=13,30, p=0.054)$. The correlation coefficients between fallypride binding potential and WCST are shown in Figure 1. Healthy volunteers tended to show positive correlations indicating better performance with higher binding potential, in contrast to patients with schizophrenia who showed negative or very low correlations. We examined the distribution of the 52 correlations (4 WCST scores vs 13 binding potential regional values).

The mean z-transformed correlation coefficients between the 4 WCST variables and the 13 brain regions were 0.12 for healthy volunteers and -0.40 for patients with schizophrenia (see Table 2). The Kolmogorov-Smirnov test showed the distributions to be different (healthy/patient permutation, $D=0.90, p<0.001$ ) and the Welch $\mathrm{t}$ test (healthy/patient permutation, $p<0.001$ ). The Jenkins-derived statistic was exactly 0.001 . The difference between healthy volunteers and patients for the binding potential in the medial dorsal nucleus was significant (healthy volunteers $\mathrm{BP}=3.06, \mathrm{sd}=0.31$, patients $\mathrm{BP}=2.77, \mathrm{sd}=0.33, p=0.0049, \mathrm{df}=40.2, \mathrm{t}=2.98)$. In the dorsolateral frontal-limbic-temporal region set (Figure 2), the BP values were significantly lower in patients (Hotelling T2 $=45.5, \mathrm{df}=14,29, p=0.049$ ). The correlations between the 4 WCST scores and 14 ROI (56) were more positive in healthy volunteers and this difference was significant by Welch $\mathrm{t}$-test $(\mathrm{t}=6.05, \mathrm{df}=109$, permutation analysis, $p<0.001)$ and Kolmogorov-Smirnov $(D=0.46$, permutation analysis, $p=0.049)$. The distribution of correlations had a low kurtosis $(-0.54$ 
$p=0.034$ simulation) value in healthy volunteers) and 0.34 in patients (kurtosis not significantly different from zero). The distribution of correlations in healthy volunteers had a skew of 0.14 but in patients it was $-0.80(p<0.001)$. Exploratory voxel-by-voxel maps or the correlation between the WCST perseverative errors and binding potential were calculated, and the Fisher $z$ test for difference between correlation coefficients were applied (Figure 3). Better performance in the WCST perseverative errors was associated with higher binding potential in the frontal cortex and right temporal lobe and lower binding in caudate. The reverse pattern was observed in patients with schizophrenia. Patients with schizophrenia showed a negative correlation between binding potential and perseverative errors in the frontal cortex, and positive correlations in the striatum.

\subsection{D2/D3 receptor binding and the CVLT}

Patients with schizophrenia underperformed on the CVLT (Table 1) compared with healthy volunteers and this was also confirmed across all nine scores (Hotelling T2, $F(9,34)=2.57$, $p=0.022$ ). Patients with schizophrenia tended to have lower binding potential for all 13 regions. This was significant on univariate testing for the medial dorsal nucleus of the thalamus ( $t=2.98$, $\mathrm{df}=40.2, p=.004)$, the lateral dorsal nucleus $(t=1.94, \mathrm{df}=39.9, p=.05)$, and medial geniculate $(t=2.26, \mathrm{df}=36.1, p=.03)$ but not multivariate testing (Hotelling T2, $F(13,30)=1.17, p=0.35)$.

The correlation coefficients between the fronto-striatal-thalamic fallypride binding potential set and CVLT are shown in Figure 4. Healthy volunteers showed positive correlations indicating better performance with higher binding potential, in contrast to patients who showed negative or very low correlations.

We examined the distribution of the 117 correlations (9 CVLT scores vs. 13 binding potential regional values). The mean z-transformed correlation coefficients between the 9 CVLT 
variables and the 13 brain regions were 0.12 in healthy volunteers' and -0.08 in patients. The regional distribution of 18F-Fallypride correlations was significantly different across groups. The Kolmogorov-Smirnoff $\mathrm{D}$ test confirmed a distribution difference in both groups, $D=0.54, p=$ 3.664e-15 permutational analysis $(D=0.54, p=0.046)$ (see Figure 4$)$. The Welch t-test showed a significant difference between groups $(t=9.03 \mathrm{df}=230.3, p=2.2 \mathrm{e}-16$ and permutation test was $p=0.025)$. We found the healthy sample (117 binding potential vs CVLT score correlations) to have significant negative skew $(-0.7$, permutation with random numbers, $\mathrm{p}<0.001)$ in comparison with the patient group (0.08) and permutation analysis with patients and healthy found this at the 0.096 level. The healthy sample had greater positive kurtosis $(0.65$, permutation with random numbers, $p=0.034)$ than patients with schizophrenia (-0.15) (permutation analysis with patients and healthy found this at the 0.098 level). The correlations between the dorsolateral frontallimbic-temporal lobe correlation coefficient data set $(9$ CVLT $\times 14$ ROI=126 r) showed similar results with healthy volunteers having more positive correlations (mean: 0.16$)$ than patients (mean: -0.07) (Kolmogorov-Smirnov $\mathrm{D}=0.69$, permutation $p=0.002$, Welch $\mathrm{t}$-test, $\mathrm{t}=15.66$, $\mathrm{df}=248.2, p<0.001)$ (See Figure 5). Exploratory voxel-by-voxel maps or the correlation between the learning slope and binding potential were calculated, and the Fisher $z$ test for difference between correlation coefficients was applied (Figure 6). Successful CVLT performance, as defined by high learning over successive trials, was associated with high binding potential in the frontal lobes and in the cingulate gyrus in healthy volunteers. The reverse pattern was seen in patients with schizophrenia. This is the opposite of the expected effect since patients with schizophrenia are hypothesized to have high dopamine in the synapse but were observed to have low binding potential in these areas in contrast to healthy volunteers. Significant clusters of Fisher $z$-tests are presented in Table 3. 


\subsection{Model-based analysis}

We examined the similarity of our data to the ventral striatal F-DOPA uptake data of (Deserno et al., 2015). A Fisher's Z comparison between the $r$ correlation map of CVLT shortdelay vs. binding potential (analogue of model-free) and the $r$ correlation map of WCST categories achieved(analogue of model-based) showed a strong negative correlation in the ventral striatum but not the dorsal striatum. This was consistent with the positive correlation shown on the difference scores (Deserno et al., 2015). Patients with schizophrenia showed a positive correlation in the dorsal striatum, suggesting an entirely different dopaminergic mechanism involving the two hypothetical models (see Figure 7). 


\section{Discussion}

To our knowledge, this study represents the first comprehensive investigation of the association between both striatal and extrastriatal D2/D3 receptor binding potential with executive function and verbal learning in medication-naïve ( 5 drug-free) patients with schizophrenia. The most important findings of this study are five-fold. Firstly, we found a positive correlation between binding potential and task performance on the WCST and CVLT in healthy volunteers. Secondly, we found lower or negative correlations between binding potential and task performance in patients with schizophrenia. Third, we found greater numbers of neuropsychological task performance vs binding potential outliers among correlation coefficients in healthy volunteers. Fourth, patients with schizophrenia had more negative and near zero correlations between performance and binding potential. Fifth, in line with model-based systems theory, we found a significantly more positive correlation between binding potential and short delay performance (model-free) in the ventral striatum in healthy subjects than the correlation between binding potential and WCST categories (model-based) .

We found less dopaminergic function (high binding potential) associated with good performance in healthy controls. This was consistent with previous studies investigating D2/D3 receptor binding during a stop-signal task $(\mathrm{n}=21)$ (Ghahremani et al., 2012), and the study of Vernaleken and colleagues who reported a correlation of 0.25 between caudate nucleus $18 \mathrm{~F}$ FDOPA (net blood brain clearance) and WCST total errors (Vernaleken et al., 2007), close to our correlation of 0.27 in healthy volunteers and higher than our correlation in patients $(r=-0.28)$. Similarly, Hsieh et al. found a negative correlation $(r=-0.44)$ between striatal dopamine transporter (DAT) availability with perseverative errors in healthy subjects (good performance 
and low availability) (Hsieh et al., 2010) consistent with our finding of a positive correlation (good performance and high BP, $r=0.27$ ) in healthy volunteers, and a negative correlation in schizophrenia $(r=-0.28)$ between dopamine binding and WCST. An 18F-F-DOPA uptake study reported a negative correlation between dopamine uptake (striatal $\mathrm{K}_{\mathrm{i}}$ ) and WCST perseveration error (good performance is low uptake) in schizophrenia (n=6) (Meyer-Lindenberg et al., 2002), a finding in the opposite direction. Consistent with previous drug-naïve or never-medicated schizophrenia studies (Andersen et al., 2013; Brickman et al., 2004; He et al., 2013; Hill et al., 2004; Zhang et al., 2012), we found significant differences between schizophrenia and healthy volunteers on short and long-delay recall. The reported negative correlations between binding potential and performance on the CVLT and positive correlations in healthy comparisons are similar in pattern to those observed with the WCST.

We examined the theoretical approach of Deserno and colleagues (Deserno et al., 2015) which evaluated the cognitive mapping of habitual and goal directed systems, termed "modelfree" and "model-based". Their description of the concepts of model-free and model bases appear analogous to CVLT short delay (models of learning based on past rewards) and WCST categories achieved (map possible actions to their potential outcomes). They found a positive correlation was between the ventral striatum F-DOPA and the difference in model-free and model based performance. Consistent with this, with binding potential, we found that the correlation between CVLT performance and binding potential in the ventral striatum but not dorsal striatum was significantly more negative than the correlation between WCST and binding potential (sign reversed since binding potential and F-DOPA uptake have related physiology but in opposite directions). 
In a novel spatial attention activation 18F-Fallypride study using the Posner Flanker task (LaBerge and Buchsbaum, 1990; Posner, 1980), we showed an association between higher dopamine release and better performance in healthy volunteers (Christian et al., 2006). In the current study, we speculate that the better performance association with low binding potential might represent an adaptation of the disturbed dopamine circuitry connections. Our findings of negative correlation between extrastriatal binding potential and high performance only in patients with schizophrenia is consistent with the recent findings of Norbak-Emig et al. (NorbakEmig et al., 2016) who assessed epidepride binding before and after antipsychotic treatment and cognitive performance using the Rapid Visual Processing (signal detection A') test from the Cambridge Neuropsychological Test Automated Battery. They interpreted their data as suggesting "that blockade of frontal D2/D3 receptors by antipsychotics may worsen some cognitive domains possibly by compromising the optimal prefrontal network functioning, which is required for cognitive performance, motivation and learning" (Norbak-Emig et al., 2016). Our findings are in line with a SPECT study on first-episode drug-naïve patients with schizophrenia, reporting an association of $\mathrm{D} 2 / \mathrm{D} 3$ receptor binding potential on cognitive measures of executive function (Stockings of Cambridge), attention (rapid visual information processing), and setshifting ability (intra-extra dimensional) using the CANTAB test battery (Fagerlund et al., 2013). The study also reported that the frontal D2/D3 availability was more highly represented during task performance in schizophrenia patients compared with healthy subjects. Taken together, these studies suggest that higher dopaminergic function may be associated with worse WCST performance in healthy individuals, diminishing flexibility, and that there may be the opposite association in schizophrenia. Indeed, perseveration in schizophrenia may be related to failure to generate or shift to a new plan, which may be consistent with both WCST perseverative scores 
and CVLT learning slope and semantic scores (Lanser et al., 2002). However, tasks with high attentional unvarying demands may show the benefits of greater dopaminergic function. Our findings support the view that striatal dopamine might diminish in cognitive flexibility but assist attention and plays an important mechanistic role in mediating learned associations between cues and environmental interpretation.

We found relatively low correlation coefficients between performance and binding potential spread across many regions of interest. This is consistent with a widespread frontotemporal-occipital activation network during the WCST, a finding that resonates with connectivity studies suggesting a disorganization of brain anatomical networks in schizophrenia where there is a loss of hierarchical organization in which high clustering of high-degree nodes resulting in inefficient axonal wiring (Bassett et al., 2008). Computational studies demonstrate that broad dispersed modular networks are involved in dealing with high processing demands from the environment (Bullmore and Sporns, 2012). The WCST evaluates an individual's ability to modify incorrect strategies in relation to environmental feedback and inhibit prepotent responses. Our findings suggest that schizophrenia may have dysfunctional modular organization networks (Yu et al., 2012) which set them apart from healthy subjects in meeting the higherorder cognitive processing demands, despite relevant target sources, the frontal and temporal cortices, showing high correlates with WCST task performance.

Evidence suggests that schizophrenia may involve a dysfunction in the frontotemporal network that is responsive to executive demands in healthy subjects (Ragland et al., 1998). The findings indicate an inability to adapt to changes in ongoing cues and feedback during task performance. Evidence from personality studies have shown that impulsivity and inhibitory control predict (Fino et al., 2014) or are associated with (Leshem and Glicksohn, 2012) the 
WCST. Although deficits in WCST performance have been consistently reported (FatourosBergman et al., 2014), our earlier study of never-medicated patients (Brickman et al., 2004), and the current study sample showed no significant difference in scores but differences in functional neuroanatomy. This implies that the differences we found were not merely related to a difference in task performance but to differences in the relationship between regional binding potential and cognitive performance. We found a striking difference in striatal and extrastriatal binding potential between groups; good performance on the WCST was associated with high binding potential in healthy volunteers, whereas medication-naive patients with schizophrenia showed a negative correlation with lower binding potential associated with better performance. The pattern suggests widespread brain activation across fronto-temporal-occipital regions in schizophrenia during WCST task performance while healthy volunteers show enhanced binding potential in more 'relevant' anatomical regions based on executive task demands. Evidence suggests that performance on executive function tasks may be exacerbated by impulsivity traits which are associated with the failure to inhibit habitual responses and switch strategies necessary based on demands imposed by environmental feedback. A 18F-Fallypride PET study in healthy volunteers with amphetamine found that reduced D2/D3 receptor binding was associated with higher levels of impulsivity (Buckholtz et al., 2010). Executive function tasks demand greater reliance on higher-order conceptualization, strategy and the integration of feedback which requires a high level of cognitive flexibility to rapidly modify a response to environmental cues. Patients with schizophrenia show difficulties in rule switching ('stuck in set tendency') despite receiving negative feedback suggesting an extreme form of perseverative tendency (Crider, 1997; Schneider and Asarnow, 1987). The widespread correlation between fronto-temporal-and occipital region binding potential and WCST performance in medication-naive patients with 
schizophrenia may represent impulsivity trait features and a compensatory mechanism for meeting the demands of the task. The medication-naïve patient sample may also be bias in containing patients with better executive function than those remaining unhospitalised without medication. This matching in task performance between healthy volunteers and the patient group may be advantageous in holding performance level equal and allowing differences in functional anatomy unconfounded by performance to be evident. Ragland and coworkers (Ragland et al., 1998) used selected poor performance volunteers on the WCST to test whether activation pattern differences between volunteers and patients with schizophrenia were only due to performance deficits rather than changes in functional anatomy.

Limitations of this study include the sample size, but the difficulties of obtaining nevermedicated patients with schizophrenia cannot be underestimated. The sample size was sufficient to demonstrate reduced binding potential for the thalamus and other regions and to demonstrate reduced performance in the CVLT. The patient selection may be biased by refusing medication and remaining un-hospitalized for much or all of their illness and in a few cases refusing medication after a single dose. However the fact that they did not have their receptor numbers and sensitivity affected by chronic medication is a major advantage of this sample. A second limitation is the separation between scanner binding potential while resting correlated with prescan neuropsychological evaluation. Any task performance correlation must then reflect trait features of dopaminergic levels of activation and trait features of cognitive performance. The alteration of dopamine function in schizophrenia is hypothesized to be genetic and stable and the test-retest reliability of the WCST over time has been documented (Seidman et al., 1991): these stabilities support our approach. A third limitation is the possibility that patients with schizophrenia had more head movement during the scan acquisition and therefore had less 
reliable scans. Three studies report that head motion is no greater in patients with schizophrenia than healthy volunteers (Buchsbaum et al., 2007; Mayer et al., 2007; Yoo et al., 2005). Power and coworkers report that head motion produces higher intercorrelations of structures close together and lower correlations of structures that are far apart as expected from the effect of the blurring of adjacent voxels (Power et al., 2012) . However, examination of our binding potential data indicates that adjacent regions (e.g. BA 20 and 21) showed Fisher z scores close to zero when healthy and patients were compared, which is inconsistent with the head-motion contribution to group differences in this data set.

Ballard and coworkers (Ballard et al., 2015) reported the WCST proportion of nonperseverative errors to be positively correlation (although non-significant) with striatal D2/D3 receptor availability in methamphetamine users, while a negative correlation was found for nonuser controls. In contrast, a similar analysis on the number of non-perseverative errors in our sample showed a positive correlation with striatal D2/D3 binding potential in healthy volunteers, and a negative correlation (all non-significant) in schizophrenia. The inconsistency in findings may reflect differences in dopamine physiology between unmedicated methamphetamine users and patients with schizophrenia, or related to the significant weakness between the different correlations.

Healthy volunteers showed increased skewness and negative kurtosis in the $r$ distributions. The activation imaging of the WCST shows small areas of activation, and large areas of lack of significant activation. Thus one would expect some few regions with high correlations with performance and more regions with low or zero correlations, a skewed distribution. If patients with schizophrenia had poorer activation or activation more variable in brain region between subjects then diminished skewness would be expected, consistent with our 
observation. However we also observed significant negative kurtosis, an indicator of a distribution that is flatter. This combination of skewness and negative kurtosis suggests that healthy individuals have not only more high value correlations but also more distributed low value correlations: a pattern of several areas of high correlation combined with diffuse participation of dopamine binding across wide brain areas. Patients with schizophrenia were not significantly skewed or kurtotic, suggesting heterogeneous dopamine deployment and/or weak, variable performance.

The link between neuroleptic medication use and its influence on executive function task performance has been controversial. Some studies have reported significant improvements in the WCST following olanzapine medication (Borkowska et al., 2002; Harvey et al., 2003; Rossi et al., 1997), both olanzapine and risperidone (Bilder et al., 2002), or amphetamine challenge (Slifstein et al., 2010), while others have not (Green et al., 2002; Purdon et al., 2000), including a follow-up study involving WCST assessment at four time points following risperidone and conventional neuroleptic medication use in schizophrenia (Remillard et al., 2005). We suggested above that tasks requiring greater flexibility such as the WCST might benefit from low dopaminergic activity while tasks requiring low flexibility but continuous attention might benefit from higher dopaminergic activity. A beneficial effect of haloperidol on WCST and a detrimental effect of haloperidol on reaction time were noted in a four week random assignment medication trial (Gallhofer et al., 2007).

Our findings in this study reinforce the concept of distributed influence of dopamine on performance involving both striatal and extrastriatal regions. These influences are trait-like in nature extending across cognitive tasks in healthy subjects. Patients with schizophrenia show relationships between performance and binding potential that are anomalous and likely 
heterogeneous, leaving a disturbed distribution of regional binding potential versus performance correlations. Our findings are also consistent with the widely interconnected network patterns observed in fMRI connectivity studies rather than with a restricted network of several small, localized, and specialized neural loci.

\section{Conflict of Interest}

All authors declare that they have no conflicts of interest.

\section{Contributors}

Prof Buchsbaum and Dr Lehrer designed the study. Dr Lehrer recruited, diagnosed and supervised the data collection. Dr Doninger, Dr Christian, and Dr Merrill quantified, co-registered and obtained ROI files. Dr Vyas, Dr Lehrer and Prof Buchsbaum managed the literature searches and analyses. Prof Mukherjee developed [F-18]Fallypride and supervised the synthesis. Prof Buchsbaum and Dr Vyas undertook the statistical analysis. Dr Vyas wrote the first draft of the manuscript. All authors contributed to and have approved the final manuscript.

\section{Acknowledgments}

We are grateful to all patients and healthy volunteers who participated in our study. Dr. Vyas is supported by a Winston Churchill Travelling Fellowship by the Winston Churchill Memorial Trust. Major funding was provided by the Kettering Health Network Foundation Additional support was provided by a grant to Dr. Buchsbaum, Anatomy and function of the thalamus in schizophrenia MH60023 and by the Department of Psychiatry, University of California, San Diego. The support of the United States Air Force, Air Force Research Laboratory (AFRL/HEOP), Air Force Materiel Command, under cooperative agreement F33615-98-2-6002, for use of imaging resources, is gratefully acknowledged. The project was approved by the IRBs of the Kettering Health Network, Wright State University and Mount Sinai School of Medicine. 


\section{References}

Addington, J., Brooks, B.L., Addington, D., 2003. Cognitive functioning in first episode psychosis: initial presentation. Schizophrenia research 62(1-2), 59-64.

Aleman, A., Hijman, R., de Haan, E.H.F., Kahn, R.S., 1999. Memory impairment in schizophrenia: A meta-analysis. American Journal of Psychiatry 156(9), 1358-1366.

Alexander, G.E., DeLong, M.R., Strick, P.L., 1986. Parallel organization of functionally segregated circuits linking basal ganglia and cortex. Annual review of neuroscience 9, 357-381.

Alexander, M.P., Stuss, D.T., Fansabedian, N., 2003. California Verbal Learning Test: performance by patients with focal frontal and non-frontal lesions. Brain 126(Pt 6), 1493 1503.

Andersen, R., Fagerlund, B., Rasmussen, H., Ebdrup, B.H., Aggernaes, B., Gade, A., Oranje, B., Glenthoj, B., 2013. The influence of impaired processing speed on cognition in firstepisode antipsychotic-naive schizophrenic patients. Eur Psychiat 28(6), 332-339.

Andreasen, N., 1997. The role of the thalamus in schizophrenia. Canadian Journal of Psychiatry 42, 27-33.

Andreasen, N., Flaum, M., Arndt, S., 1992. The Comprehensive Assessment of Symptoms and History (CASH): An instrument for assessing diagnosis and psychopathology. Arch Gen Psychiatry 49, 615-623.

Andreasen, N., O'Leary, D., Flaum, M., Nopoulos, P., Watkins, G., Boles-Ponto, L., Hichwa, R., 1997. Hypofrontality in schizophrenia: distributed dysfunctional circuits in neurolepticnaive patients. The Lancet 349, 1730-1734.

Baldo, J.V., Delis, D., Kramer, J., Shimamura, A.P., 2002. Memory performance on the California Verbal Learning Test-II: findings from patients with focal frontal lesions. J Int Neuropsychol Soc 8(4), 539-546.

Ballard, M.E., Mandelkern, M.A., Monterosso, J.R., Hsu, E., Robertson, C.L., Ishibashi, K., Dean, A.C., London, E.D., 2015. Low Dopamine D2/D3 Receptor Availability is Associated with Steep Discounting of Delayed Rewards in Methamphetamine Dependence. The international journal of neuropsychopharmacology / official scientific journal of the Collegium Internationale Neuropsychopharmacologicum 18(7), pyu119.

Barcelo, F., Sanz, M., Molina, V., Rubia, F.J., 1997. The Wisconsin Card Sorting Test and the assessment of frontal function: a validation study with event-related potentials. Neuropsychologia 35(4), 399-408.

Bassett, D.S., Bullmore, E., Verchinski, B.A., Mattay, V.S., Weinberger, D.R., MeyerLindenberg, A., 2008. Hierarchical organization of human cortical networks in health and schizophrenia. The Journal of neuroscience : the official journal of the Society for Neuroscience 28(37), 9239-9248.

Berman, K.F., Ostrem, J.L., Randolph, C., Gold, J., Goldberg, T.E., Coppola, R., Carson, R.E., Herscovitch, P., Weinberger, D.R., 1995. Physiological activation of a cortical network during performance of the Wisconsin Card Sorting Test: a positron emission tomography study. Neuropsychologia 33(8), 1027-1046.

Bilder, R.M., Goldman, R.S., Robinson, D., Reiter, G., Bell, L., Bates, J.A., Pappadopulos, E., Willson, D.F., Alvir, J.M., Woerner, M.G., Geisler, S., Kane, J.M., Lieberman, J.A., 
2000. Neuropsychology of first-episode schizophrenia: initial characterization and clinical correlates. The American journal of psychiatry 157(4), 549-559.

Bilder, R.M., Goldman, R.S., Volavka, J., Czobor, P., Hoptman, M., Sheitman, B., Lindenmayer, J.P., Citrome, L., McEvoy, J., Kunz, M., Chakos, M., Cooper, T.B., Horowitz, T.L., Lieberman, J.A., 2002. Neurocognitive effects of clozapine, olanzapine, risperidone, and haloperidol in patients with chronic schizophrenia or schizoaffective disorder. The American journal of psychiatry 159(6), 1018-1028.

Borkowska, A., Araszkiewicz, A., Rajewski, A., Rybakowski, J.K., 2002. Risperidone treatment of schizophrenia: improvement in psychopathology and neuropsychological tests. Neuropsychobiology 46(2), 85-89.

Bressler, S.L., Menon, V., 2010. Large-scale brain networks in cognition: emerging methods and principles. Trends in cognitive sciences 14(6), 277-290.

Brickman, A.M., Buchsbaum, M.S., Bloom, R., Bokhoven, P., Paul-Odouard, R., Haznedar, M., Dahlman, K., Hazlett, E.A., Aronowitz, J., Heath, D., Shihabuddin, L., in press. Neuropsychological functioning in first-break, never-medicated adolescents with psychosis. Journal of Nervous and Mental Disease.

Brickman, A.M., Buchsbaum, M.S., Bloom, R., Bokhoven, P., Paul-Odouard, R., Haznedar, M.M., Dahlman, K.L., Hazlett, E.A., Aronowitz, J., Heath, D., Shihabuddin, L., 2004. Neuropsychological functioning in first-break, never-medicated adolescents with psychosis. The Journal of nervous and mental disease 192(9), 615-622.

Brickman, A.M., Buchsbaum, M.S., Shihabuddin, L., Hazlett, E.A., Borod, J.C., Mohs, R.C., 2003. Striatal size, glucose metabolic rate, and verbal learning in normal aging. Brain Res Cogn Brain Res 17(1), 106-116.

Brix, G., Zaers, J., Adam, L.E., Bellemann, M.E., Ostertag, H., Trojan, H., Haberkorn, U., Doll, J., Oberdorfer, F., Lorenz, W.J., 1997. Performance evaluation of a whole-body PET scanner using the NEMA protocol. National Electrical Manufacturers Association. J Nucl Med 38(10), 1614-1623.

Buchsbaum, B.R., Greer, S., Chang, W.L., Berman, K.F., 2005. Meta-analysis of neuroimaging studies of the Wisconsin card-sorting task and component processes. Human brain mapping 25(1), 35-45.

Buchsbaum, M.S., Buchsbaum, B.R., Hazlett, E.A., Haznedar, M., Newmark, R., Tang, C., Hof, P.R., 2007. Relative Glucose Metabolic Rate Higher in White Matter in Schizophrenia. American Journal of Psychiatry 164, 1072-1081.

Buchsbaum, M.S., Christian, B.T., Lehrer, D.S., Narayanan, T.K., Shi, B., Mantil, J., Kemether, E., Oakes, T.R., Mukherjee, J., 2006. D2/D3 dopamine receptor binding with [F18]fallypride in thalamus and cortex of patients with schizophrenia. Schizophrenia research 85(1-3), 232-244.

Buchsbaum, M.S., Hazlett, E.A., Haznedar, M.M., Spiegel-Cohen, J., Wei, T.C., 1999. Visualizing fronto-striatal circuitry and neuroleptic effects in schizophrenia. Acta Psychiatr Scand Suppl 395, 129-137.

Buckholtz, J.W., Treadway, M.T., Cowan, R.L., Woodward, N.D., Li, R., Ansari, M.S., Baldwin, R.M., Schwartzman, A.N., Shelby, E.S., Smith, C.E., Kessler, R.M., Zald, D.H., 2010. Dopaminergic network differences in human impulsivity. Science 329(5991), 532.

Buckley, P.F., 2005. Neuroimaging of schizophrenia: structural abnormalities and pathophysiological implications. Neuropsychiatr Dis Treat 1(3), 193-204. 
Bullmore, E., Sporns, O., 2012. The economy of brain network organization. Nature reviews. Neuroscience 13(5), 336-349.

Christian, B.T., Lehrer, D.S., Shi, B., Narayanan, T.K., Strohmeyer, P.S., Buchsbaum, M.S., Mantil, J.C., 2006. Measuring dopamine neuromodulation in the thalamus: using [F18]fallypride PET to study dopamine release during a spatial attention task. Neuroimage 31(1), 139-152.

$\backslash$ Cohen, J. Statistical Power Analysis for the Behavioral Sciences, Academic Press, New York, 1969).

Crider, A., 1997. Perseveration in schizophrenia. Schizophrenia bulletin 23(1), 63-74.

Cunningham, V.J., Hume, S.P., Price, G.R., Ahier, R.G., Cremer, J.E., Jones, A.K., 1991. Compartmental analysis of diprenorphine binding to opiate receptors in the rat in vivo and its comparison with equilibrium data in vitro. J Cereb Blood Flow Metab 11(1), 1-9.

Davidson, L.L., Heinrichs, R.W., 2003. Quantification of frontal and temporal lobe brainimaging findings in schizophrenia: a meta-analysis. Psychiatry Res 122(2), 69-87.

Delis, D., Kramer, J., Kaplan, E., Ober, B., 1987. The California Verbal Learning Test. Psychological Corporation, New York.

Delis, D.C., Kramer, J.H., Kaplan, E., Ober, B. , 2000. CVLT-II, California Verbal Learning Test, adult version manual. The Psychological Corporation, New York.

Deserno, L., Huys, Q.J., Boehme, R., Buchert, R., Heinze, H.J., Grace, A.A., Dolan, R.J., Heinz, A., Schlagenhauf, F., 2015. Ventral striatal dopamine reflects behavioral and neural signatures of model-based control during sequential decision making. Proc Natl Acad Sci U S A 112(5), 1595-1600.

Dickinson, D., Harvey, P.D., 2009. Systemic hypotheses for generalized cognitive deficits in schizophrenia: a new take on an old problem. Schizophrenia bulletin 35(2), 403-414.

Ellison-Wright, I., Glahn, D.C., Laird, A.R., Thelen, S.M., Bullmore, E., 2008. The anatomy of first-episode and chronic schizophrenia: an anatomical likelihood estimation metaanalysis. The American journal of psychiatry 165(8), 1015-1023.

Ettinger, U., Corr, P.J., Mofidi, A., Williams, S.C., Kumari, V., 2013. Dopaminergic basis of the psychosis-prone personality investigated with functional magnetic resonance imaging of procedural learning. Front Hum Neurosci 7, 130.

Fagerlund, B., Pagsberg, A.K., Hemmingsen, R.P., 2006. Cognitive deficits and levels of IQ in adolescent onset schizophrenia and other psychotic disorders. Schizophrenia research 85(1-3), 30-39.

Fagerlund, B., Pinborg, L.H., Mortensen, E.L., Friberg, L., Baare, W.F., Gade, A., Svarer, C., Glenthoj, B.Y., 2013. Relationship of frontal D(2/3) binding potentials to cognition: a study of antipsychotic-naive schizophrenia patients. The international journal of neuropsychopharmacology / official scientific journal of the Collegium Internationale Neuropsychopharmacologicum 16(1), 23-36.

Farde, L., Suhara, T., Nyberg, S., Karlsson, P., Nakashima, Y., Hietala, J., Halldin, C., 1997. A PET-study of [11C]FLB 457 binding to extrastriatal D2-dopamine receptors in healthy subjects and antipsychotic drug-treated patients. Psychopharmacology 133(4), 396-404.

Fatouros-Bergman, H., Cervenka, S., Flyckt, L., Edman, G., Farde, L., 2014. Meta-analysis of cognitive performance in drug-naive patients with schizophrenia. Schizophrenia research 158(1-3), 156-162. 
Fino, E., Melogno, S., Iliceto, P., D'Aliesio, S., Pinto, M.A., Candilera, G., Sabatello, U., 2014. Executive functions, impulsivity, and inhibitory control in adolescents: A structural equation model. Adv Cogn Psychol 10(2), 32-38.

Fioravanti, M., Carlone, O., Vitale, B., Cinti, M.E., Clare, L., 2005. A meta-analysis of cognitive deficits in adults with a diagnosis of schizophrenia. Neuropsychol Rev 15(2), 73-95.

First, M.B., Spitzer, R.L., Gibbon, M., Williams, J.B., 1996. Structured clinical interview for DSM-IV. American Psychiatric Association, Washington, DC.

Gallhofer, B., Jaanson, P., Mittoux, A., Tanghoj, P., Lis, S., Krieger, S., 2007. Course of recovery of cognitive impairment in patients with schizophrenia: a randomised doubleblind study comparing sertindole and haloperidol. Pharmacopsychiatry 40(6), 275-286.

Ghahremani, D.G., Lee, B., Robertson, C.L., Tabibnia, G., Morgan, A.T., De Shetler, N., Brown, A.K., Monterosso, J.R., Aron, A.R., Mandelkern, M.A., Poldrack, R.A., London, E.D., 2012. Striatal dopamine $\mathrm{D}(2) / \mathrm{D}(3)$ receptors mediate response inhibition and related activity in frontostriatal neural circuitry in humans. The Journal of neuroscience : the official journal of the Society for Neuroscience 32(21), 7316-7324.

Glahn, D.C., Laird, A.R., Ellison-Wright, I., Thelen, S.M., Robinson, J.L., Lancaster, J.L., Bullmore, E., Fox, P.T., 2008. Meta-analysis of gray matter anomalies in schizophrenia: application of anatomic likelihood estimation and network analysis. Biological psychiatry 64(9), 774-781.

Glenthoj, B.Y., Mackeprang, T., Svarer, C., Rasmussen, H., Pinborg, L.H., Friberg, L., Baare, W., Hemmingsen, R., Videbaek, C., 2006. Frontal Dopamine D(2/3) Receptor Binding in Drug-Naive First-Episode Schizophrenic Patients Correlates with Positive Psychotic Symptoms and Gender. Biological psychiatry.

Gonzalez-Hernandez, J.A., Pita-Alcorta, C., Cedeno, I., Bosch-Bayard, J., Galan-Garcia, L., Scherbaum, W.A., Figueredo-Rodriguez, P., 2002. Wisconsin Card Sorting Test synchronizes the prefrontal, temporal and posterior association cortex in different frequency ranges and extensions. Human brain mapping 17(1), 37-47.

Green, M.F., Kern, R.S., Braff, D.L., Mintz, J., 2000. Neurocognitive deficits and functional outcome in schizophrenia: are we measuring the "right stuff"? Schizophrenia bulletin 26(1), 119-136.

Green, M.F., Marder, S.R., Glynn, S.M., McGurk, S.R., Wirshing, W.C., Wirshing, D.A., Liberman, R.P., Mintz, J., 2002. The neurocognitive effects of low-dose haloperidol: a two-year comparison with risperidone. Biological psychiatry 51(12), 972-978.

Harvey, P.D., Green, M.F., McGurk, S.R., Meltzer, H.Y., 2003. Changes in cognitive functioning with risperidone and olanzapine treatment: a large-scale, double-blind, randomized study. Psychopharmacology 169(3-4), 404-411.

Hazlett, E.A., Buchsbaum, M.S., Zhang, J., Newmark, R.E., Glanton, C.F., Zelmanova, Y., Haznedar, M.M., Chu, K.W., Nenadic, I., Kemether, E.M., Tang, C.Y., New, A.S., Siever, L.J., 2008. Frontal-striatal-thalamic mediodorsal nucleus dysfunction in schizophrenia-spectrum patients during sensorimotor gating. NeuroImage.

He, Z., Deng, W., Li, M., Chen, Z., Jiang, L., Wang, Q., Huang, C., Collier, D.A., Gong, Q., Ma, X., Zhang, N., Li, T., 2013. Aberrant intrinsic brain activity and cognitive deficit in firstepisode treatment-naive patients with schizophrenia. Psychol Med 43(4), 769-780.

Heaton, R.K., \& PAR Staff, 2003. Wisconsin Card Sorting Test: Computer Version 4, Research Edition (WCST: CV4). Psychological Assessment Resources, Odessa. 
Heinrichs, R.W., Zakzanis, K.K., 1998. Neurocognitive deficit in schizophrenia: A quantitative review of the evidence. Neuropsychology 12(3), 426-445.

Hill, S.K., Beers, S.R., Kmiec, J.A., Keshavan, M.S., Sweeney, J.A., 2004. Impairment of verbal memory and learning in antipsychotic-naive patients with first-episode schizophrenia. Schizophrenia research 68(2-3), 127-136.

Hill, S.K., Ragland, J.D., Gur, R.C., Gur, R.E., 2001. Neuropsychological differences among empirically derived clinical subtypes of schizophrenia. Neuropsychology 15(4), 492-501.

Hsieh, P.C., Yeh, T.L., Lee, I.H., Huang, H.C., Chen, P.S., Yang, Y.K., Chiu, N.T., Lu, R.B., Liao, M.H., 2010. Correlation between errors on the Wisconsin Card Sorting Test and the availability of striatal dopamine transporters in healthy volunteers. J Psychiatr Neurosci 35(2), 90-94.

Ichise, M., Toyama, H., Innis, R.B., Carson, R.E., 2002. Strategies to improve neuroreceptor parameter estimation by linear regression analysis. J Cereb Blood Flow Metab 22(10), 1271-1281.

Ito, H., Kodaka, F., Takahashi, H., Takano, H., Arakawa, R., Shimada, H., Suhara, T., 2011. Relation between presynaptic and postsynaptic dopaminergic functions measured by positron emission tomography: implication of dopaminergic tone. The Journal of neuroscience : the official journal of the Society for Neuroscience 31(21), 7886-7890.

Ito, M., Doya, K., 2009. Validation of decision-making models and analysis of decision variables in the rat basal ganglia. The Journal of neuroscience : the official journal of the Society for Neuroscience 29(31), 9861-9874.

Joyce, J.N., Janowsky, A., Neve, K.A., 1991. Characterization and distribution of [125I] epidepride binding to dopamine D2 receptors in basal ganglia and cortex of human brain. J Pharmacol Exp Ther 257(3), 1253-1263.

Kay, S.R., Fiszbein, A., Opler, L.A., 1987. The positive and negative syndrome scale (PANSS) for schizophrenia. Schizophrenia bulletin 13(2), 261-276.

Kessler, R.M., Whetsell, W.O., Ansari, M.S., Votaw, J.R., de Paulis, T., Clanton, J.A., Schmidt, D.E., Mason, N.S., Manning, R.G., 1993. Identification of extrastriatal dopamine D2 receptors in post mortem human brain with [125I]epidepride. Brain Res 609(1-2), 237243.

Kessler, R.M., Woodward, N.D., Riccardi, P., Li, R., Ansari, M.S., Anderson, S., Dawant, B., Zald, D., Meltzer, H.Y., 2009. Dopamine D2 receptor levels in striatum, thalamus, substantia nigra, limbic regions, and cortex in schizophrenic subjects. Biol Psychiat 65(12), 1024-1031.

Kumari, V., Antonova, E., Geyer, M.A., 2008. Prepulse inhibition and "psychosis-proneness" in healthy individuals: an fMRI study. Eur Psychiatry 23(4), 274-280.

LaBerge, D., Buchsbaum, M.S., 1990. Positron emission tomographic measurements of pulvinar activity during an attention task. The Journal of neuroscience : the official journal of the Society for Neuroscience 10(2), 613-619.

Lanser, M.G., Berger, H.J., Ellenbroek, B.A., Cools, A.R., Zitman, F.G., 2002. Perseveration in schizophrenia: failure to generate a plan and relationship with the psychomotor poverty subsyndrome. Psychiatry Res 112(1), 13-26.

Lehrer, D., Christian, B.T., Kirbas, C., Chiang, M., Sidhu, S., Short, H., Wang, B., Shi, B., K-W, C., MS, B., 2010a. 18F-Fallypride binding potential in patients with schizophrenia compared to healthy controls. Schizophrenia research 122, 43-52. 
Lehrer, D.S., Christian, B.T., Kirbas, C., Chiang, M., Sidhu, S., Short, H., Wang, B., Shi, B., Chu, K.W., Merrill, B., Buchsbaum, M.S., 2010b. 18F-fallypride binding potential in patients with schizophrenia compared to healthy controls. Schizophrenia research 122(13), 43-52.

Lehrer, D.S., Christian, B.T., Mantil, J., Murray, A.C., Buchsbaum, B.R., Oakes, T.R., Byne, W., Kemether, E.M., Buchsbaum, M.S., 2005. Thalamic and prefrontal FDG uptake in never medicated patients with schizophrenia. The American journal of psychiatry 162(5), 931938.

Leshem, R., Glicksohn, J., 2012. A Critical Look at the Relationship Between Impulsivity and Decision-Making in Adolescents: Are They Related or Separate Factors? Dev Neuropsychol 37(8), 712-731.

Li, C.S.R., 2004. Do schizophrenia patients make more perseverative than non-perseverative errors on the Wisconsin Card Sorting Test? A meta-analytic study. Psychiatry Research 129(2), 179-190.

Liang, P., Wang, Z., Yang, Y., Jia, X., Li, K., 2011. Functional disconnection and compensation in mild cognitive impairment: evidence from DLPFC connectivity using resting-state fMRI. PloS one 6(7), e22153.

Logan, J., Fowler, J.S., Volkow, N.D., Wang, G.J., Ding, Y.S., Alexoff, D.L., 1996. Distribution volume ratios without blood sampling from graphical analysis of PET data. J Cereb Blood Flow Metab 16(5), 834-840.

Mayer, A.R., Franco, A.R., Ling, J., Canive, J.M., 2007. Assessment and quantification of head motion in neuropsychiatric functional imaging research as applied to schizophrenia. $\mathrm{J}$ Int Neuropsychol Soc 13(5), 839-845.

McClellan, J., Prezbindowski, A., Breiger, D., McCurry, C., 2004. Neuropsychological functioning in early onset psychotic disorders. Schizophrenia research 68(1), 21-26.

Meyer-Lindenberg, A., Miletich, R.S., Kohn, P.D., Esposito, G., Carson, R.E., Quarantelli, M., Weinberger, D.R., Berman, K.F., 2002. Reduced prefrontal activity predicts exaggerated striatal dopaminergic function in schizophrenia. Nat Neurosci 5(3), 267-271.

Milner, B., 1963. Effects of Different Brain Lesions on Card sorting: the role of the frontal lobes. Archives of Neurology 9, 90-100.

Mohamed, S., Paulsen, J.S., O'Leary, D., Arndt, S., Andreasen, N., 1999. Generalized cognitive deficits in schizophrenia: a study of first-episode patients. Arch Gen Psychiatry 56(8), 749-754.

Mukherjee, J., Christian, B.T., Dunigan, K.A., Shi, B., Narayanan, T.K., Satter, M., Mantil, J., 2002. Brain imaging of 18F-fallypride in normal volunteers: Blood analysis, distribution, test-retest studies, and preliminary assessment of sensitivity to aging effects on dopamine D-2/D-3 receptors. Synapse 46(3), 170-188.

Mukherjee, J., Yang, Z.Y., Das, M.K., Brown, T., 1995. Fluorinated benzamide neuroleptics--III. Development of (S)-N-[(1-allyl-2-pyrrolidinyl)methyl]-5-(3-[18F]fluoropropyl)-2, 3dimethoxybenzamide as an improved dopamine D-2 receptor tracer. Nucl Med Biol 22(3), 283-296.

Norbak-Emig, H., Ebdrup, B.H., Fagerlund, B., Svarer, C., Rasmussen, H., Friberg, L., Allerup, P.N., Rostrup, E., Pinborg, L.H., Glenthoj, B.Y., 2016. Frontal D2/3 Receptor Availability in Schizophrenia Patients Before and After Their First Antipsychotic Treatment: Relation to Cognitive Functions and Psychopathology. The international 
journal of neuropsychopharmacology / official scientific journal of the Collegium Internationale Neuropsychopharmacologicum.

Nyhus, E., Barcelo, F., 2009. The Wisconsin Card Sorting Test and the cognitive assessment of prefrontal executive functions: a critical update. Brain Cogn 71(3), 437-451.

Oie, M., Rund, B.R., 1999. Neuropsychological deficits in adolescent-onset schizophrenia compared with attention deficit hyperactivity disorder. American Journal of Psychiatry 156(8), 1216-1222.

Overall, J.E., 1972. The Brief Psychiatric Rating Sclae in Psychopharmacology. University of Texas Galveston, Research Psychometric Laboratory Reports.

Overall, J.E., 1976. The Brief Psychiatric Rating Scale, NIMH Rockville, Maryland.

Patel, N.H., Vyas, N.S., Puri, B.K., Nijran, K.S., Al-Nahhas, A., 2010. Positron emission tomography in schizophrenia: a new perspective. J Nucl Med 51(4), 511-520.

Paulsen, J.S., Heaton, R.K., Sadek, J.R., Perry, W., Delis, D.C., Braff, D., Kuck, J., Zisook, S., Jeste, D.V., 1995. The nature of learning and memory impairments in schizophrenia. J Int Neuropsychol Soc 1(1), 88-99.

Posner, M.I., 1980. Orienting of Attention. Q J Exp Psychol 32(Feb), 3-25.

Power, J.D., Barnes, K.A., Snyder, A.Z., Schlaggar, B.L., Petersen, S.E., 2012. Spurious but systematic correlations in functional connectivity MRI networks arise from subject motion. Neuroimage 59(3), 2142-2154.

Purdon, S.E., Jones, B.D., Stip, E., Labelle, A., Addington, D., David, S.R., Breier, A., Tollefson, G.D., 2000. Neuropsychological change in early phase schizophrenia during 12 months of treatment with olanzapine, risperidone, or haloperidol. The Canadian Collaborative Group for research in schizophrenia. Arch Gen Psychiatry 57(3), 249-258.

Ragland, J.D., Gur, R.C., Glahn, D.C., Censits, D.M., Smith, R.J., Lazarev, M.G., Alavi, A., Gur, R.E., 1998. Frontotemporal cerebral blood flow change during executive and declarative memory tasks in schizophrenia: a positron emission tomography study. Neuropsychology 12(3), 399-413.

Reichenberg, A., Caspi, A., Harrington, H., Houts, R., Keefe, R.S., Murray, R.M., Poulton, R., Moffitt, T.E., 2010. Static and dynamic cognitive deficits in childhood preceding adult schizophrenia: a 30-year study. The American journal of psychiatry 167(2), 160-169.

Remillard, S., Pourcher, E., Cohen, H., 2005. The effect of neuroleptic treatments on executive function and symptomatology in schizophrenia: a 1-year follow up study. Schizophrenia research 80(1), 99-106.

Riehemann, S., Volz, H.P., Stutzer, P., Smesny, S., Gaser, C., Sauer, H., 2001. Hypofrontality in neuroleptic-naive schizophrenic patients during the Wisconsin Card Sorting Test--a fMRI study. European archives of psychiatry and clinical neuroscience 251(2), 66-71.

Riley, E.M., McGovern, D., Mockler, D., Doku, V.C., S, O.C., Fannon, D.G., Tennakoon, L., Santamaria, M., Soni, W., Morris, R.G., Sharma, T., 2000. Neuropsychological functioning in first-episode psychosis--evidence of specific deficits. Schizophrenia research 43(1), 47-55.

Roofeh, D., Cottone, J., Burdick, K.E., Lencz, T., Gyato, K., Cervellione, K.L., Napolitano, B., Kester, H., Anderson, B., Kumra, S., 2006. Deficits in memory strategy use are related to verbal memory impairments in adolescents with schizophrenia-spectrum disorders. Schizophrenia research 85(1-3), 201-212. 
Rossi, A., Mancini, F., Stratta, P., Mattei, P., Gismondi, R., Pozzi, F., Casacchia, M., 1997. Risperidone, negative symptoms and cognitive deficit in schizophrenia: An open study. Acta Psychiat Scand 95(1), 40-43.

Saykin, A.J., Shtasel, D.L., Gur, R.E., Kester, D.B., Mozley, L.H., Stafiniak, P., Gur, R.C., 1994. Neuropsychological Deficits in Neuroleptic Naive Patients with First-Episode Schizophrenia. Arch Gen Psychiatry 51(2), 124-131.

Schneider, S.G., Asarnow, R.F., 1987. A comparison of cognitive/neuropsychological impairments of nonretarded autistic and schizophrenic children. J Abnorm Child Psychol 15(1), 29-45.

Seidman, L.J., Pepple, J.R., Faraone, S.V., Kremen, W.S., Cassens, G., McCarley, R.W., Tsuang, M.T., 1991. Wisconsin Card Sorting Test performance over time in schizophrenia. Preliminary evidence from clinical follow-up and neuroleptic reduction studies. Schizophrenia research 5(3), 233-242.

Suhara, T., Okubo, Y., Yasuno, F., Sudo, Y., Inoue, M., Ichimiya, T., Nakashima, Y., Nakayama, K., Tanada, S., Suzuki, K., Halldin, C., Farde, L., 2002. Decreased dopamine D2 receptor binding in the anterior cingulate cortex in schizophrenia. Arch Gen Psychiatry 59(1), 25-30.

Szoke, A., Trandafir, A., Dupont, M.E., Meary, A., Schurhoff, F., Leboyer, M., 2008. Longitudinal studies of cognition in schizophrenia: meta-analysis. Br J Psychiatry 192(4), 248-257.

Takahashi, H., Higuchi, M., Suhara, T., 2006. The role of extrastriatal dopamine D2 receptors in schizophrenia. Biol Psychiat 59(10), 919-928.

Talvik, M., Nordstrom, A.L., Olsson, H., Halldin, C., Farde, L., 2003. Decreased thalamic D2/D3 receptor binding in drug-naive patients with schizophrenia: a PET study with [11C]FLB 457. The international journal of neuropsychopharmacology / official scientific journal of the Collegium Internationale Neuropsychopharmacologicum 6(4), 361-370.

Townsend, L.A., Malla, A.K., Norman, R.M., 2001. Cognitive functioning in stabilized firstepisode psychosis patients. Psychiatry Res 104(2), 119-131.

Tziortzi, A.C., Haber, S.N., Searle, G.E., Tsoumpas, C., Long, C.J., Shotbolt, P., Douaud, G., Jbabdi, S., Behrens, T.E., Rabiner, E.A., Jenkinson, M., Gunn, R.N., 2014. Connectivitybased functional analysis of dopamine release in the striatum using diffusion-weighted MRI and positron emission tomography. Cerebral cortex 24(5), 1165-1177.

Vernaleken, I., Buchholz, H.G., Kumakura, Y., Siessmeier, T., Stoeter, P., Bartenstein, P., Cumming, P., Grunder, G., 2007. 'Prefrontal' cognitive performance of healthy subjects positively correlates with cerebral FDOPA influx: an exploratory [18F]-fluoro-L-DOPAPET investigation. Human brain mapping 28(10), 931-939.

Vyas, N.S., Patel, N.H., Nijran, K.S., Al-Nahhas, A., Puri, B.K., 2011. The use of PET imaging in studying cognition, genetics and pharmacotherapeutic interventions in schizophrenia. Expert Rev Neurother 11(1), 37-51.

Vyas, N.S., Shamsi, S.A., Malhotra, A.K., Aitchison, K.J., Kumari, V., 2012. Can genetics inform the management of cognitive deficits in schizophrenia? J Psychopharmacol 26(3), 334-348.

Woods, R.P., Cherry, S.R., Mazziotta, J.C., 1992. Rapid automated algorithm for aligning and reslicing PET images. J Comput Assist Tomogr 16(4), 620-633. 
Yasuno, F., Suhara, T., Okubo, Y., Sudo, Y., Inoue, M., Ichimiya, T., Takano, A., Nakayama, K., Halldin, C., Farde, L., 2004. Low dopamine d(2) receptor binding in subregions of the thalamus in schizophrenia. The American journal of psychiatry 161(6), 1016-1022.

Yoo, S.S., Choi, B.G., Juh, R., Pae, C.U., Lee, C.U., 2005. Head motion analysis during cognitive fMRI examination: application in patients with schizophrenia. Neurosci Res 53(1), 84-90.

Zhang, X.Y., Chen, D.C., Xiu, M.H., Yang, F.D., Haile, C.N., Kosten, T.A., Kosten, T.R., 2012. Gender Differences in Never-Medicated First-Episode Schizophrenia and Medicated Chronic Schizophrenia Patients. J Clin Psychiat 73(7), 1025-1033. 
Table 1. Demographic characteristics and WCST performance in patients with schizophrenia and healthy volunteers

\begin{tabular}{|c|c|c|c|}
\hline Parameter & Schizophrenia $(n=25)$ & Healthy volunteers $(n=19)$ & Statistic \\
\hline Age, years & $31.6(12.2)$ & $29.2(9.2)$ & $t=-.73(42), p=.46$ \\
\hline $\mathrm{Sex}, \mathrm{M} / \mathrm{F}$ & $18 / 7$ & $12 / 7$ & Fisher's Exact test $=.74$ \\
\hline $\begin{array}{l}\text { Ethnicity, no. white/African American, African- } \\
\text { Jamaican/African-Caribbean/Mixed }\end{array}$ & $16 / 0 / 1 / 1 / 1$ & $22 / 3 / 0 / 0 / 0$ & $\chi^{2}=6.24, p=.18$ \\
\hline Handedness, no. right/left-handed & $23 / 2$ & $16 / 3$ & Fisher's Exact test $=.3$ \\
\hline \multicolumn{4}{|l|}{ WCST } \\
\hline Categories achieved & $5.36(1.34)$ & $5.72(0.95)$ & $t=.21, d f=34, p=.52$ \\
\hline Perseverative errors & $14.78(10)$ & $11.72(6.76)$ & $t=-1.24 . d f=36, p=0.22$ \\
\hline Shift learning & $.75(.06)$ & $.41(.71)$ & $t=-1.1, d f=35$, p. 27 \\
\hline Total errors ( $T$ score) & $50.8(11.83)$ & $50.38(10.98)$ & $t=-.11, d f=36, \mathrm{p}=.91$ \\
\hline \multicolumn{4}{|l|}{ CVLT } \\
\hline Trials $1-5$ & $43.4(9.34)$ & $54.68(9.2)$ & $t=3.79, d f=37, p=.001$ \\
\hline Short-delayed recall & $9.33(3.75)$ & $11.52(2.96)$ & $t=2.03, d f=38, p=.049$ \\
\hline Long-delayed recall & $9.57(3.75)$ & $11.89(3.36)$ & $t=2.11, d f=36, p=.042$ \\
\hline Learning slope & $1.39(.61)$ & $1.43(.56)$ & $t=.21, d f=37, p=.828$ \\
\hline Semantic cluster & $.44(1.09)$ & $1.9(2.15)$ & $t=2.69, d f=37, p=.01$ \\
\hline Recognition 'hits' & $13.65(1.59)$ & $14.73(1.28)$ & $t=2.33, d f=37, p=.026$ \\
\hline Discrimination & $2.65(.81)$ & $3.27(.72)$ & $t=2.56, d f=37, p=.016$ \\
\hline False positives & $2.8(3.04)$ & $1.26(1.88)$ & $t=.04, d f=37, p=.06$ \\
\hline Response bias & $.14(.28)$ & $.12(.34)$ & $t=.87, d f=30, p=.86$ \\
\hline Duration of illness & $204(237.3)$ & - & - \\
\hline Medication status, $n$ & $\begin{array}{l}20 \text { medication-naïve; } 5 \text { previous } \\
\text { medication use }\end{array}$ & - & - \\
\hline
\end{tabular}


Table 2. Wisconsin Card Sort score correlations with fallypride binding potential in healthy volunteers and patients with schizophrenia

\section{Healthy volunteers}

medial dorsal thatamus

lateral dorsal thalamus

pulvinar

medial geniculate thalamus

lateral geniculate thalamus

ventral caudate

ventral caudate

Brodmann area 23

Brodmann area 23

medial globus pal1idus

lateral globus pallidus

\section{Patients with schizophrenia}

medial dorsal thalamus

lateral dorsal thalamus

pulvinar

medial geniculate thalamus

lateral geniculate thatamus

ventral caudate

middle caudate

dorsal caudate

Brodmann area 23

Brodmann area 24

Brodmann area 32

medial globus pallidus

lateral globus pallidus

Fisher $\mathrm{z}$ comparison of correlations

medial dorsal thalamus

latera dorsal thalamus

pulvinar

lateral geniculate thalamus

ventral caudate

middle caudate

dorsal caudate

Brodmann area 23

Brodmann area 2

medial globus pallidus

lateral globus pallidus middle caudate

Brodmann area 24

Brodmann area

\begin{tabular}{|c|c|c|c|}
\hline $\begin{array}{l}\text { Perseverative } \\
\text { Errors } \\
-0.1267 \\
-0.3022 \\
-0.2470 \\
0.1170 \\
-0.0617 \\
0.0130 \\
-0.1067 \\
-0.2761 \\
0.0349 \\
-0.0249 \\
0.0084 \\
-0.2613 \\
-0.1656\end{array}$ & $\begin{array}{l}\text { Total } \\
\text { Correct } \\
-0.043 \\
0.047 \\
0.028 \\
-0.107 \\
-0.035 \\
0.171 \\
0.226 \\
0.219 \\
-0.021 \\
-0.020 \\
-0.093 \\
0.080 \\
0.133\end{array}$ & $\begin{array}{c}\text { Categories } \\
\text { achieved } \\
0.174 \\
0.387 \\
0.213 \\
-0.093 \\
0.136 \\
-0.169 \\
-0.054 \\
0.170 \\
-0.028 \\
0.102 \\
-0.081 \\
0.114 \\
-0.056\end{array}$ & $\begin{array}{l}-0.397 \\
-0.478 * \\
-0.316 \\
-0.261 \\
-0.354 \\
-0.087 \\
-0.147 \\
-0.253 \\
-0.175 \\
-0.215 \\
-0.114 \\
-0.123 \\
-0.138\end{array}$ \\
\hline
\end{tabular}

$\begin{array}{llll}0.363 & -0.328 & -0.60 * & 0.470 * \\ 0.351 & -0.438 * & -0.63 * & 0.512 * \\ 0.424 * & -0.445 * & -0.66 * & 0.553^{*} \\ 0.253 & -0.401 * & -0.28 & 0.099 \\ 0.437 * & -0.431 * & -0.47 & 0.327 \\ -0.047 & 0.079 & -0.16 * & -0.190 \\ 0.244 & -0.282 & -0.54 * & 0.146 \\ 0.573 * & -0.637 * & -0.74 * & 0.356 \\ 0.252 & -0.429 * & -0.46 * & 0.167 \\ 0.196 & -0.332 & -0.46 * & 0.263 \\ 0.215 & -0.257 & -0.46 * & 0.320 \\ 0.447 * & -0.403 * & -0.66 * & 0.300 \\ 0.300 & -0.277 & -0.57 * & 0.147\end{array}$

For Fisher $\mathrm{z}, *=\mathrm{p}<0.05, * *$ Bonferroni corrected $\mathrm{p}<0.05$ based on assumption of independence and $0.05 / 52$. 
Table 3. CVLT learning slope correlation with fallypride binding potential compared in healthy volunteers and patients

\begin{tabular}{|c|c|c|c|c|c|c|c|}
\hline \multirow[t]{2}{*}{$\begin{array}{l}\text { Cluster } \\
\text { number }\end{array}$} & \multicolumn{3}{|c|}{$\begin{array}{l}\text { Talairach } \\
\text { coordinates }\end{array}$} & \multirow[t]{2}{*}{$\begin{array}{c}\text { Volume } \\
\mathbf{m m}^{3}\end{array}$} & \multirow[t]{2}{*}{$\begin{array}{c}\text { Maximum } \\
\mathrm{z}\end{array}$} & \multirow[t]{2}{*}{$p$ value } & \multirow[t]{2}{*}{ Regions } \\
\hline & $\mathbf{x}$ & $\mathbf{y}$ & $\mathbf{z}$ & & & & \\
\hline 1 & 41 & -53 & -19 & 2115 & 3.87 & 0.000055 & Right Fusiform gyrus, BA 37 \\
\hline 2 & 45 & 45 & -17 & 1017 & 3.18 & 0.00074 & Right Middle, inferior frontal gyrus, BA 11, 47 \\
\hline 3 & 29 & -43 & 7 & 991 & 2.90 & 0.0019 & Right Parahippocampal gryus, hippocampus, caudate tail \\
\hline 4 & -31 & -75 & -7 & 904 & -1.69 & 0.049 & Left Middle frontal gyrus, BA 9,46 \\
\hline 5 & -39 & 45 & -17 & 773 & 2.85 & 0.0022 & Left middle frontal gyrus, BA 46, 11 \\
\hline 6 & 25 & -77 & -5 & 569 & -1.69 & 0.049 & Right Occipital lobe, lingual gyrus, BA 18, 19 \\
\hline 7 & -39 & -9 & -15 & 536 & -1.69 & 0.049 & Left Occipital lobe BA 18, 19, parahippcampal gyrus \\
\hline 8 & -35 & 23 & -31 & 417 & 2.68 & 0.0038 & Left parahippocampal gyrus, fusiform, BA 36, 20 \\
\hline 9 & 61 & -13 & -11 & 359 & 2.87 & 0.0020 & Right temporal lobe \\
\hline 10 & 11 & 41 & 17 & 265 & 2.43 & 0.0075 & $\begin{array}{l}\text { Right medial frontal gyrus, anterior cingulate, BA 9, } 32 \text {, } \\
10\end{array}$ \\
\hline
\end{tabular}

The CVLT learning slope is the rate of increase in the number of words remembered out of 16 across the five trials. Maps of the correlation coefficients between the slope values and binding potential were calculated for healthy volunteers and patients and then compared with the Fisher $\mathrm{z}$ test.

Positive $\mathrm{z}$ value indicates more positive correlation between learning slope and binding potential in healthy volunteers than patients with schizophrenia. Negative $\mathrm{z}$ value indicates more positive correlation between learning slope and binding potential in patients with schizophrenia than healthy volunteers. 
Figure 1. Correlations between WCST and fallypride binding heat maps and Fisher z comparison in patients with schizophrenia in comparison with healthy volunteers.

Healthy BP vs. WCST

$r$ value
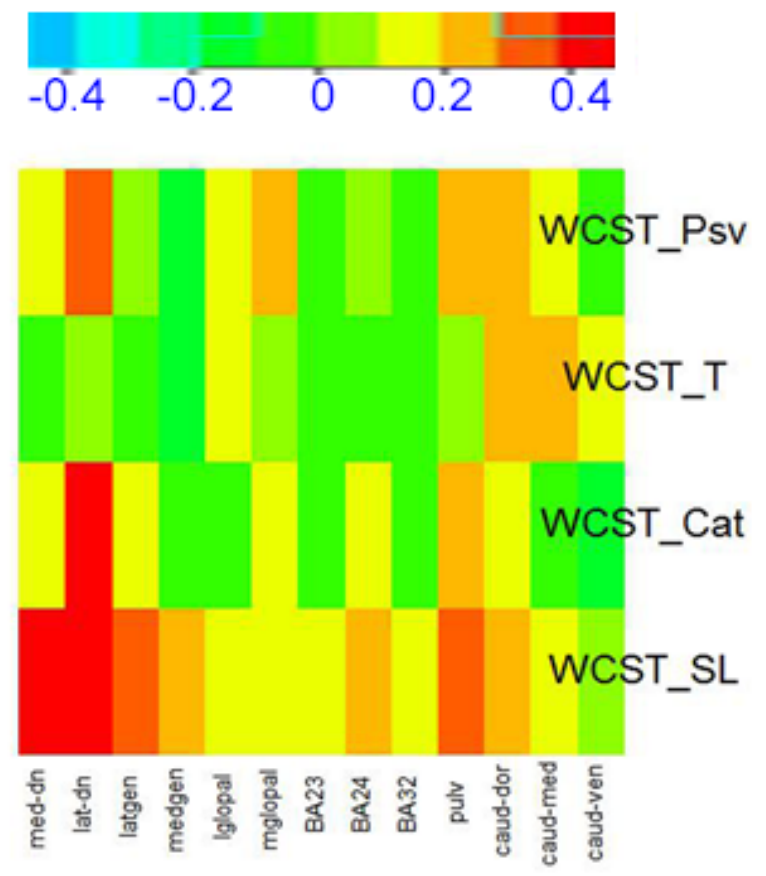

Fisher Z healthy vs schizophrenia
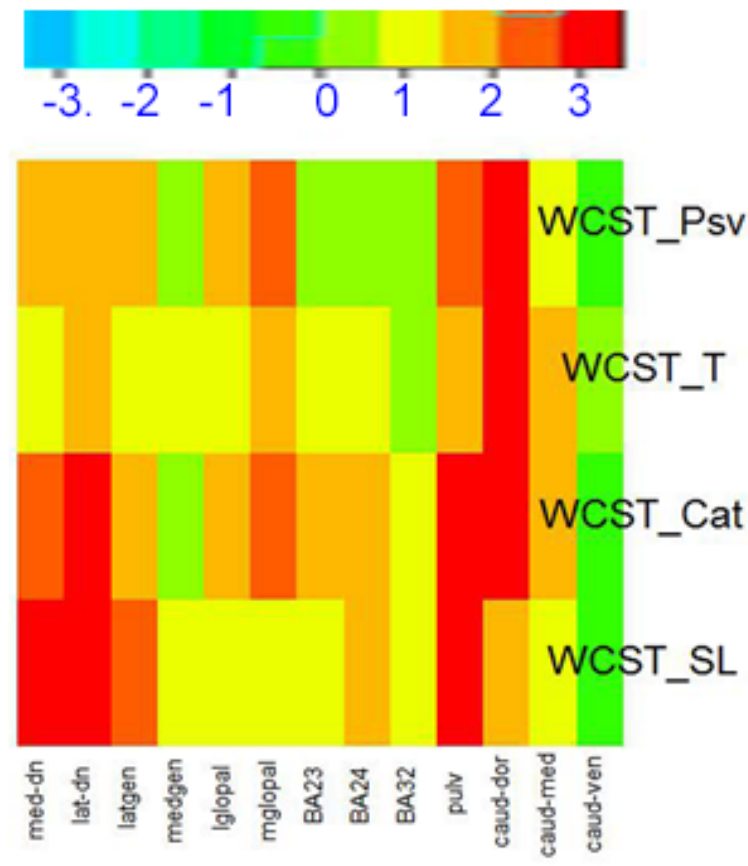

BP vs. WCST correlation histogram

Diagnosis

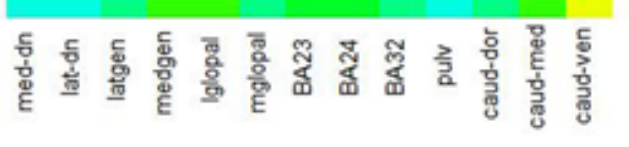

\section{Schizophrenia BP vs. WCST}
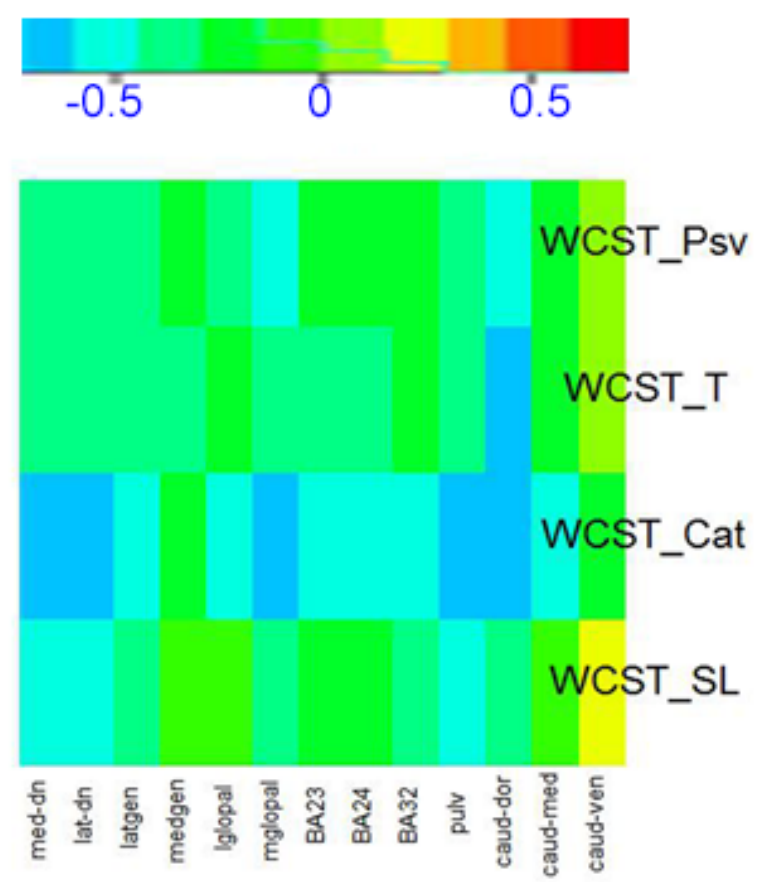

10

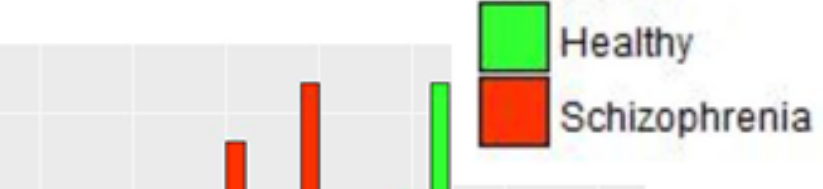

0 .

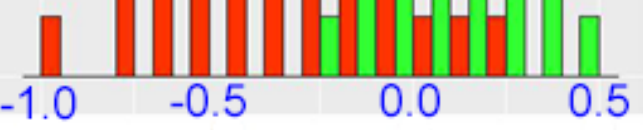

Brain region fallypride BP vs. WCST 
Heat maps show the inter-correlation of binding potential regions and WCST scores in both groups. WCST_Psv (WCST Perseveration), WCST_T (T score for total errors, higher value is good), WCST_Cat (WCST Categories achieved, higher value is good) and WCST_SL (WCST shift learning, higher value is good). The region abbreviations are defined in Table 2. Scale bar shows correlation coefficient value for the left and right panel of the upper row and the $\mathrm{z}$ value for the Fisher test of correlation coefficient differences for the left panel in the lower row. The right side of the lower row shows histograms of the subset of 52 correlations between fallypride binding ROI and WCST scale scores (4 WCST scores $\times 13$ binding potential values). The healthy volunteers show positive correlations (yellow) indicating that high binding potential (i.e. less dopamine in synapse) is associated with better performance. However patients show the reverse (blue) indicating low binding potential (i.e. more dopamine in synapse) is associated with better performance. This distribution difference is statistically confirmed (see text) and can also be seen in the histograms of the 104 correlation coefficients for fallypride binding vs CVLT scores. Healthy volunteers (green bars) show a slightly skewed positive value distribution while patient with schizophrenia (red bars) have a larger negative correlation set. Note that signs of WCST-Psv and WCST-fail set have been reversed in the upper right so that all four variables have higher values for better performance. 
Figure 2. Fronto-temporal correlations between WCST and fallypride binding heat maps and Fisher z comparison in patients with schizophrenia in comparison with healthy volunteers.

Healthy BP vs. WCST

$r$ value
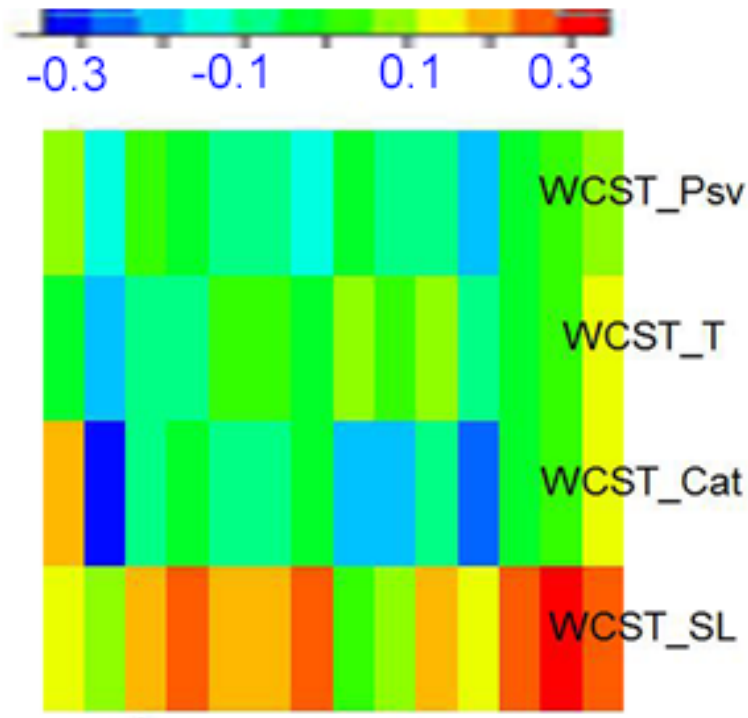

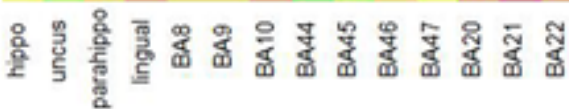

Fisher $Z$ healthy vs. schizophrenia
Schizophrenia BP vs. WCST

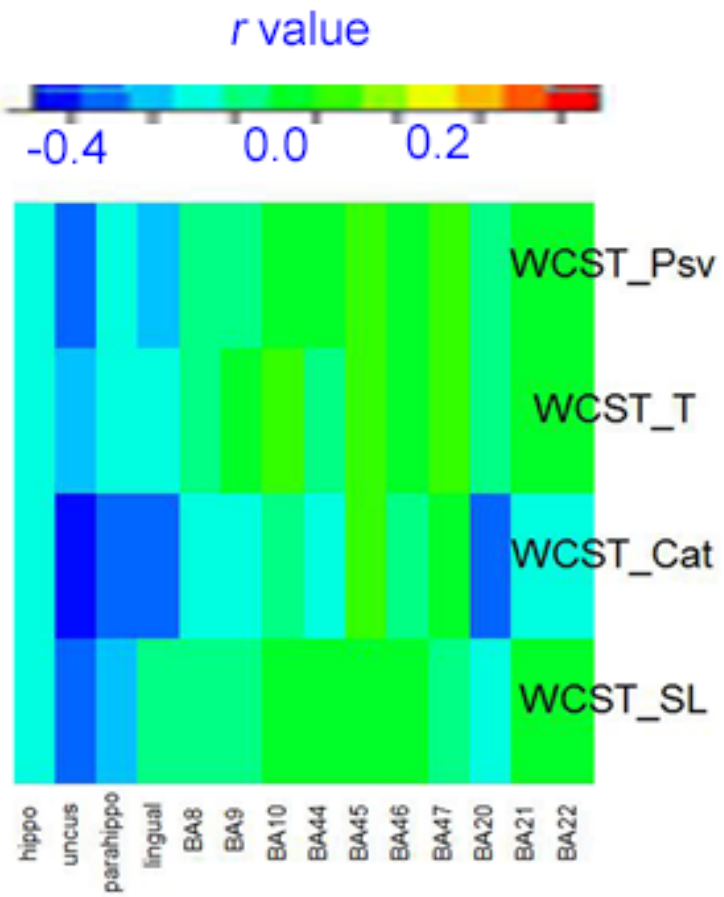

BP vs. WCST correlation histogram

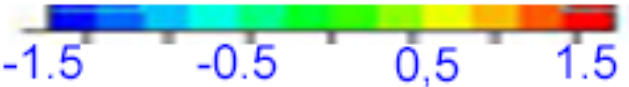

15

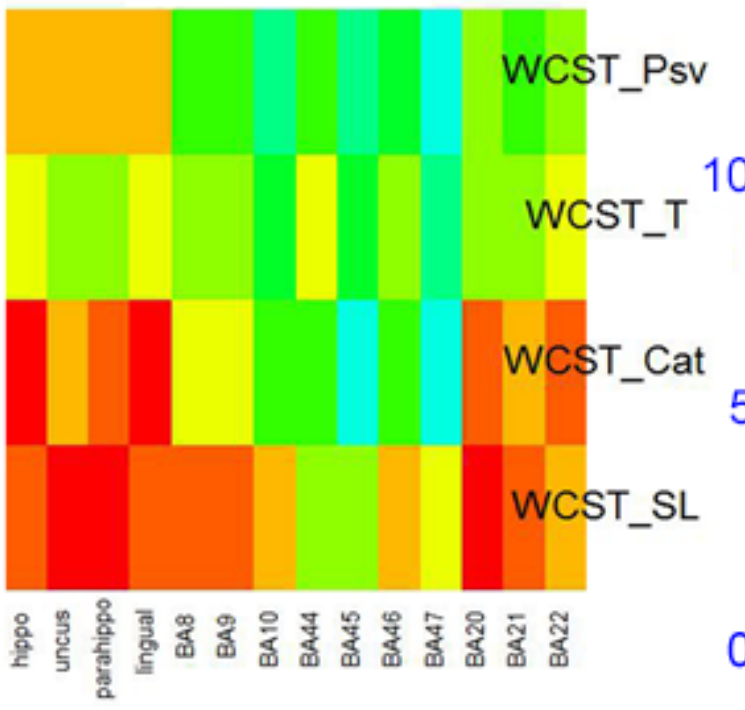

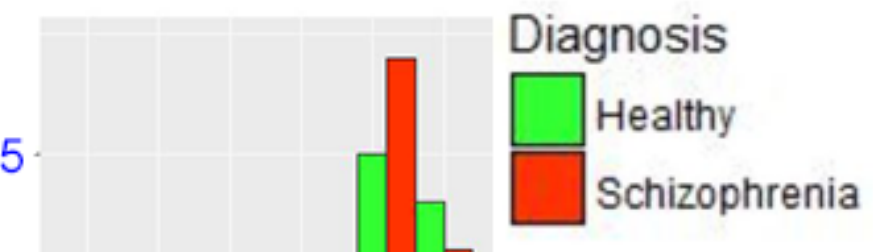

0.

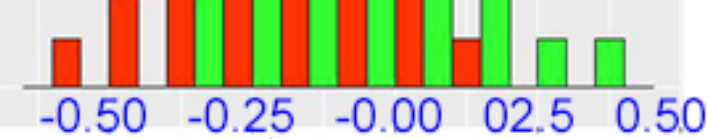

Brain region fallypride BP vs. WCST 
Heat maps show the inter-correlation of binding potential regions and WCST scores in both groups. The right side of the lower row shows histograms of the subset of 56 correlations between fallypride binding ROI and WCST scale scores (4 WCST scores $\times 14$ binding potential values). The healthy volunteers showed mostly positive correlations (red and yellow) indicating that high binding potential is associated with better performance, whereas patients with schizophrenia showed negative correlations (blue). The histograms show some overlapping correlation coefficients for fallypride binding and WCST. Abbreviations are hippocampus (hippo), parahippocampal gyrus (parahippo), and lingual gyrus (lingual). Brodmann are numbered. 
Figure 3. Correlations between WCST perseveration and fallypride binding potential

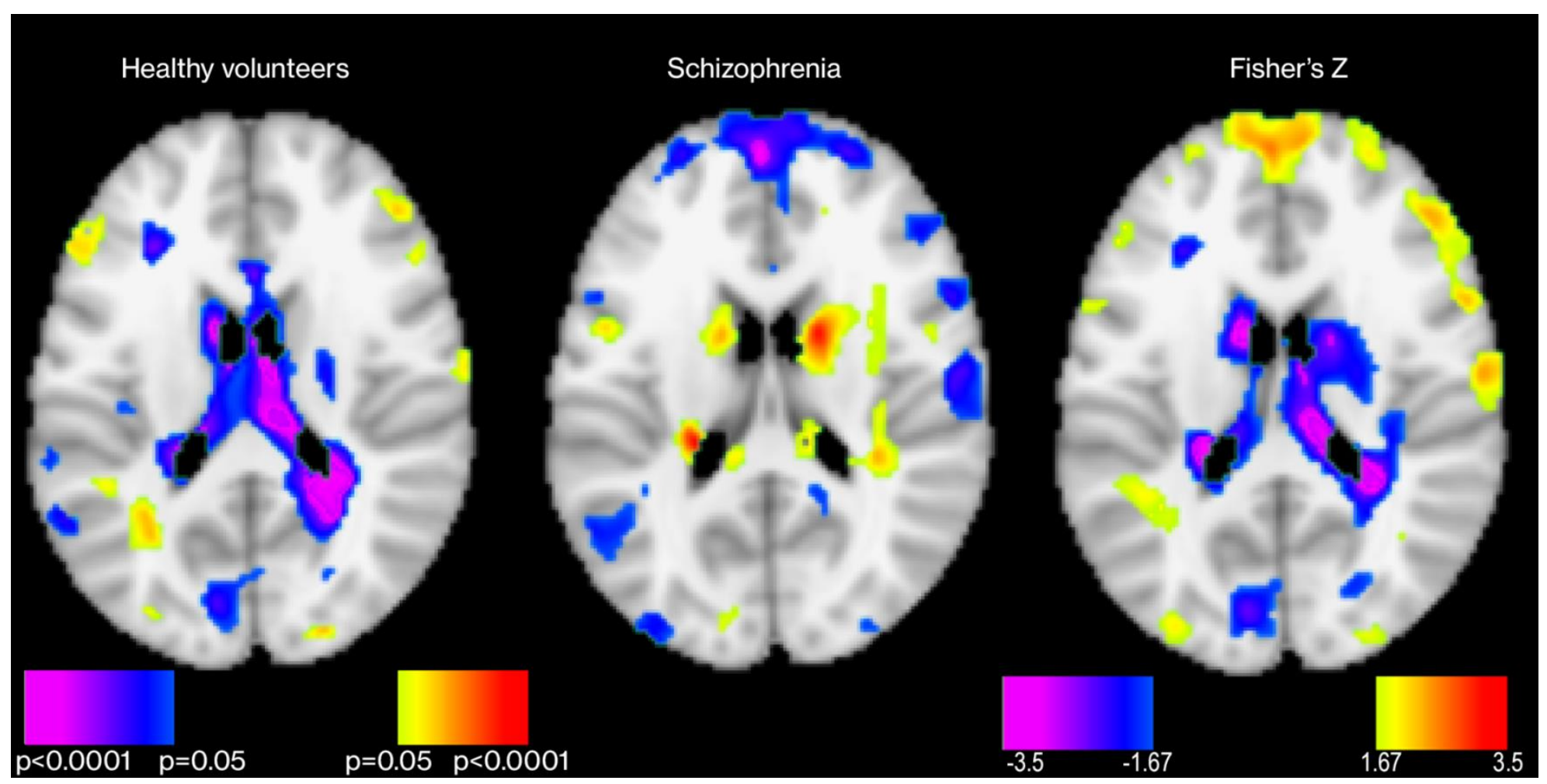

Left: Healthy volunteers' product moment correlation between WCST and fallypride binding potential. Middle: Patients with schizophrenia Right: Fisher z-test for difference in correlations with red-yellow indicating healthy volunteers correlations more positive than patients. Note that healthy volunteers with better performance have higher binding in frontal cortex and right temporal lobe and lower binding in caudate. In contrast patients tended to have negative correlations between binding potential and perseveration in the frontal cortex (high binding potential associated with low error) and positive correlations in
the
striatum
(high
binding
potential
associated
with
high
error). 
Figure 4. CVLT vs Fallypride Binding Potential Heat maps and Fisher z comparison in patients with schizophrenia in comparison with healthy volunteers.

\section{Healthy BP vs. CVLT}

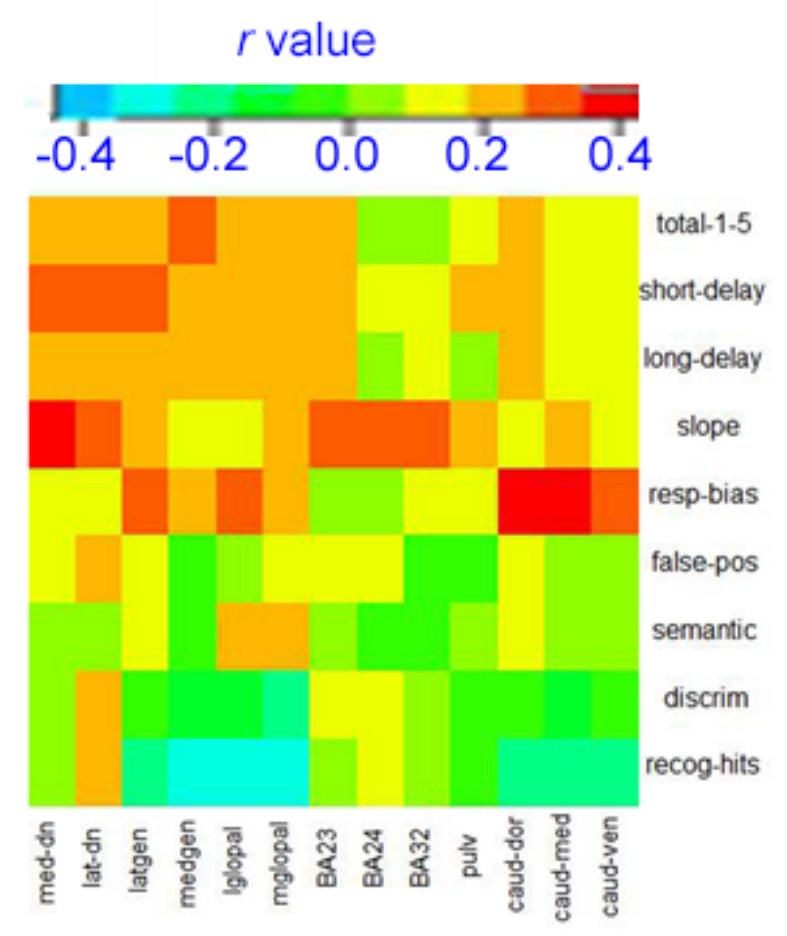

Fisher $\mathbf{Z}$ healthy vs. schizophrenia

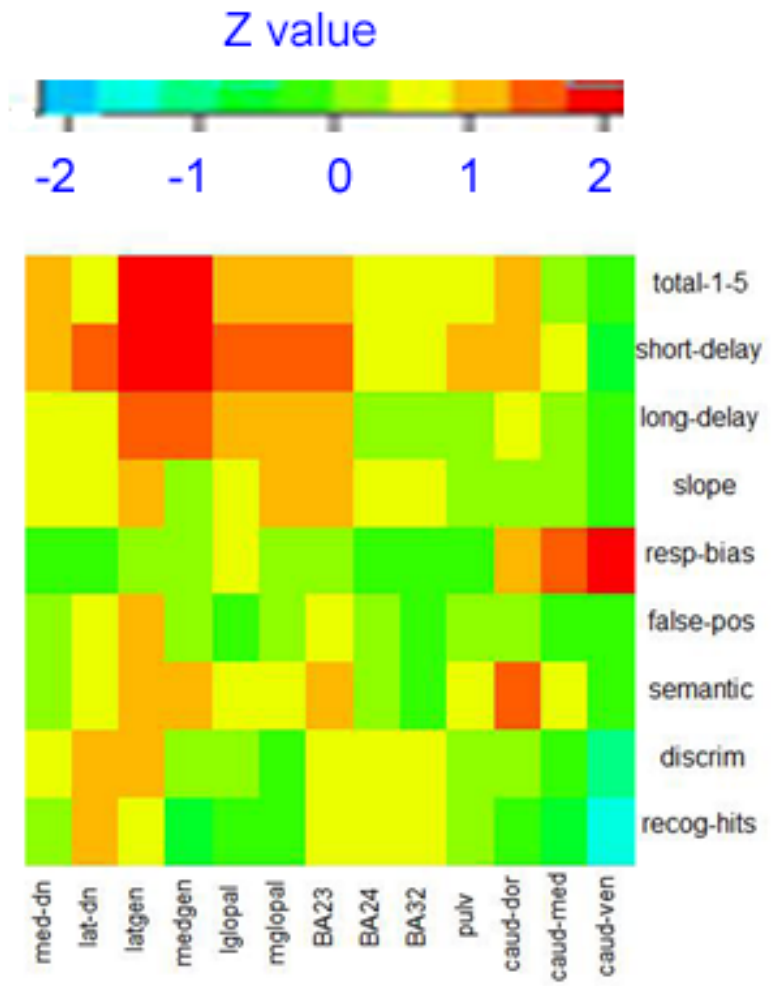

\section{Schizophrenia BP vs. CVLT}

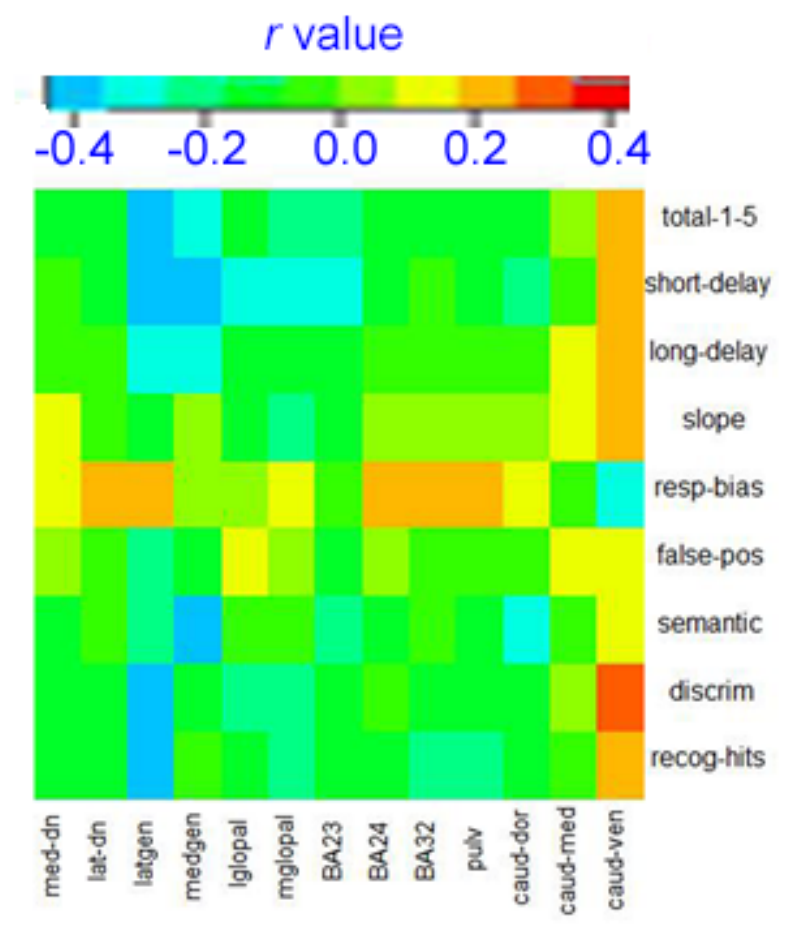

BP vs. CVLT correlation histogram

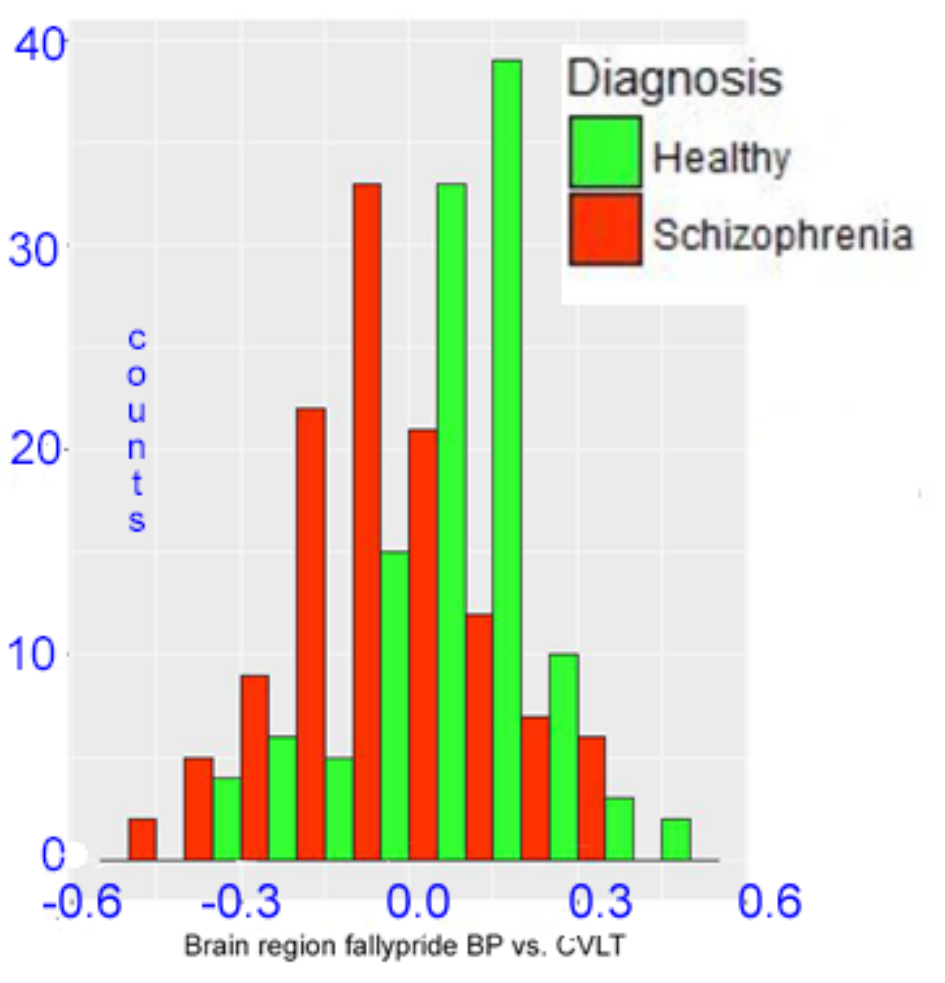


The heat maps show the inter-correlation of binding potential regions and CVLT scores in both groups. Scale bar shows correlation coefficient value for the left and right panels of the upper row and the $\mathrm{z}$ value for the Fisher test of correlation coefficient differences for the left panel in the lower row. The right panel in the lower row shows histograms of the subset of 117 correlations between fallypride binding ROI and CVLT scale scores. The difference is marked in the subset region of fallypride in the thalamus with CVLT total score (see upper left of heat maps for healthy and patients). The healthy volunteers show positive correlations (red) indicating that high binding potential (i.e. less dopamine in synapse) is associated with better performance. However patients show the reverse (blue) indicating low binding potential (i.e. more dopamine in synapse) is associated with better performance. This distribution difference is statistically confirmed (see text) and can also be seen in Fisher $\mathrm{z}$ test in the left lower row. The histograms of the 117 correlation coefficients for 13 fallypride binding regions vs. 9 CVLT $(13 \times 9=117)$ scores is shown in the lower right. Healthy volunteers (green bars) show a negative skewed value distribution while patient with schizophrenia (red bars) have a larger negative correlation sets. The abbreviations for regions are shown in Table 2. 
Figure5. CVLT vs Fronto-temporal fallypride Binding Potential Heat maps and Fisher z comparison in patients with schizophrenia in comparison with healthy volunteers. 
Healthy BP vs. CVLT
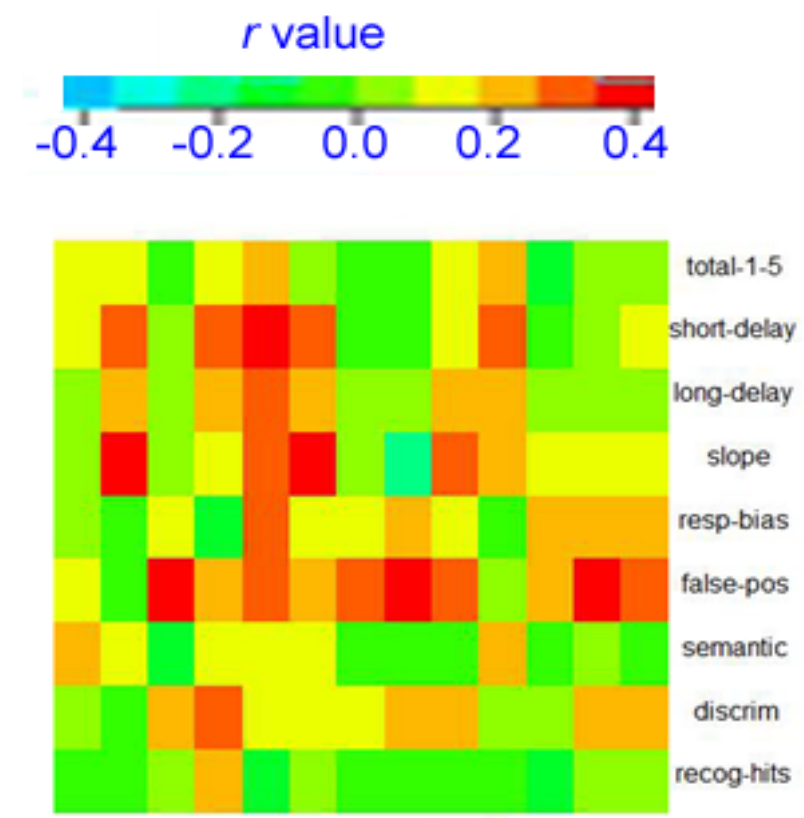

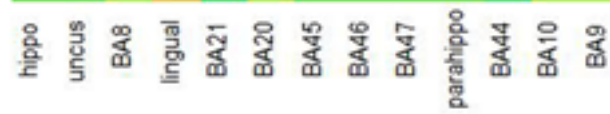

Fisher $Z$ healthy vs. schizophrenia

\section{Z value}
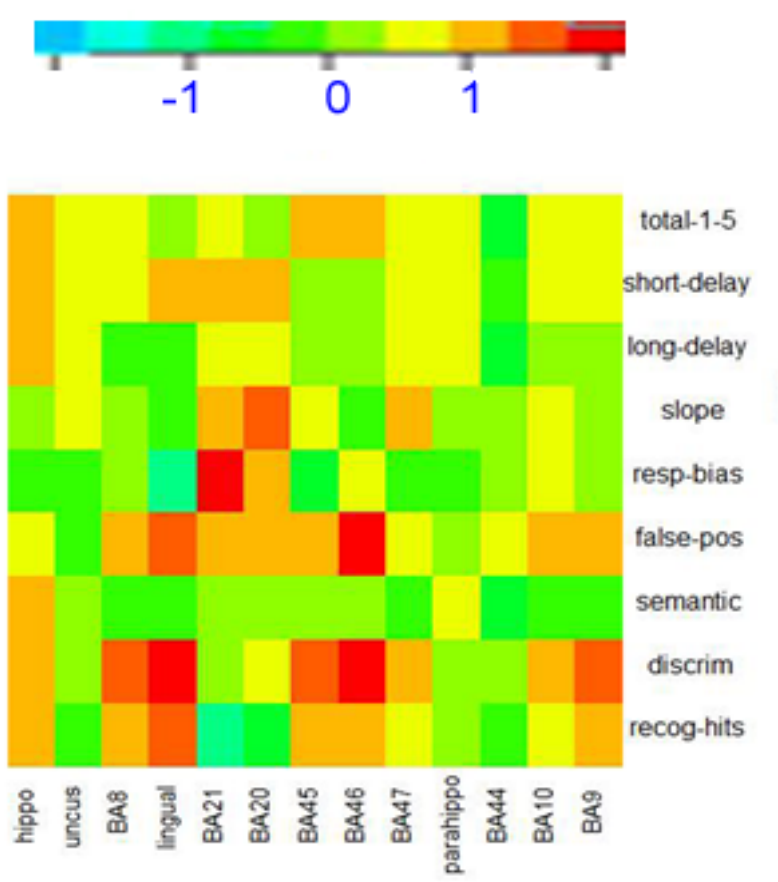

Schizophrenia BP vs. CVLT
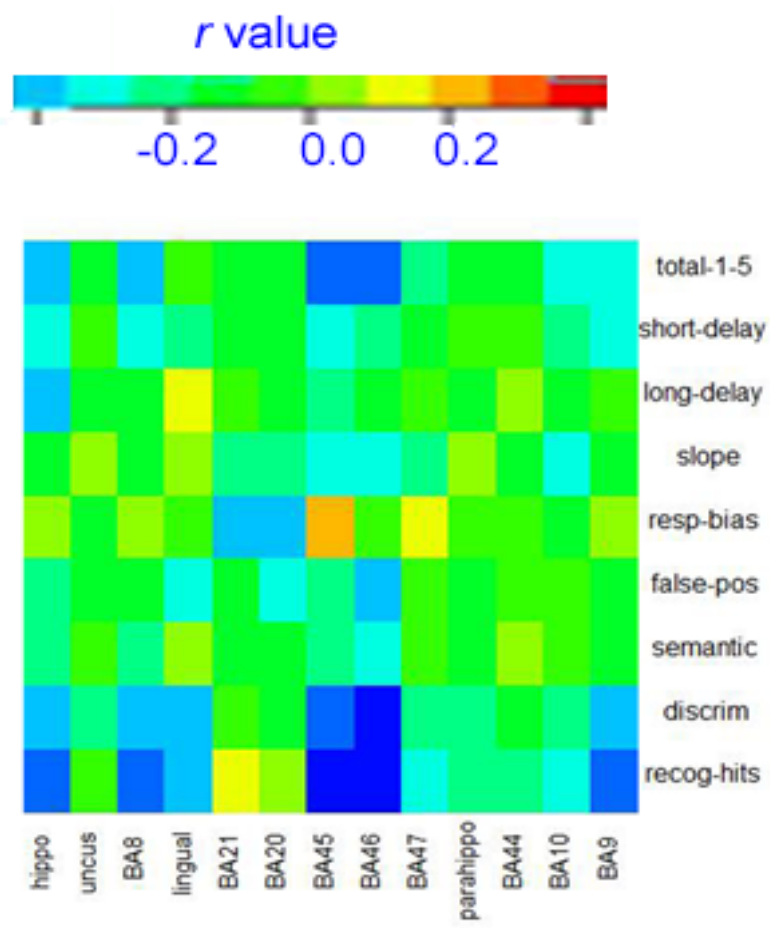

BP vs. CVLT correlation histogram

40

30

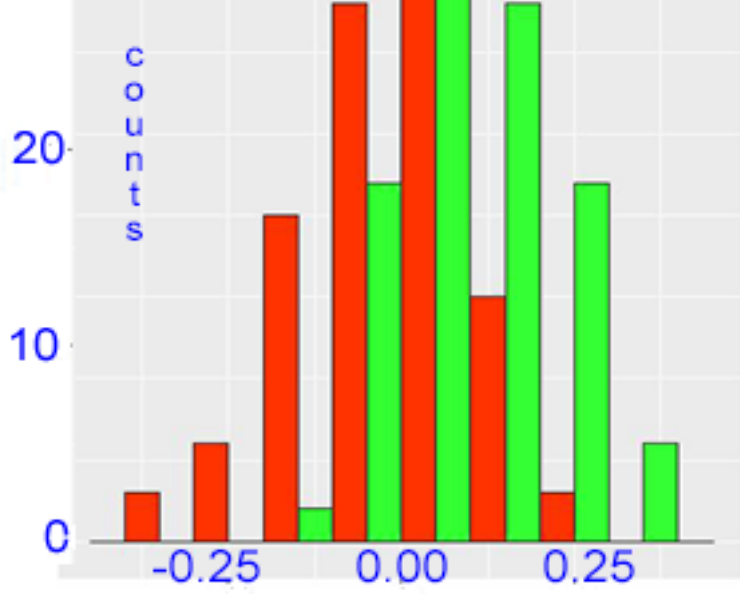

Brain region fallypride BP vs. CVLT 
The heat maps show the inter-correlation of binding potential regions and CVLT scores in both groups. Scale bar shows correlation coefficient value for the left and right panels of the upper row and the $\mathrm{z}$ value for the Fisher test of correlation coefficient differences for the left panel in the lower row. The right panel in the lower row shows histograms of the subset of 117 correlations between fallypride binding ROI and CVLT scale scores. The difference is marked in the subset region of fallypride in the temporal cortices (BA21) with CVLT total score (see upper left of heat maps for healthy and patients). The healthy volunteers show positive correlations (red) indicating that high binding potential (i.e. less dopamine in synapse) is associated with better performance. However patients show the reverse (blue) indicating low binding potential (i.e. more dopamine in synapse) is associated with better performance. This distribution difference is statistically confirmed (see text) and can also be seen in Fisher $\mathrm{z}$ test in the left lower row. Healthy volunteers (green bars) show a positive skewed value distribution while patient with schizophrenia have a larger negative correlation sets. . Abbreviations are hippocampus (hippo), parahippocampal gyrus (parahippo), and lingual gyrus (lingual). Brodmann are numbered. 


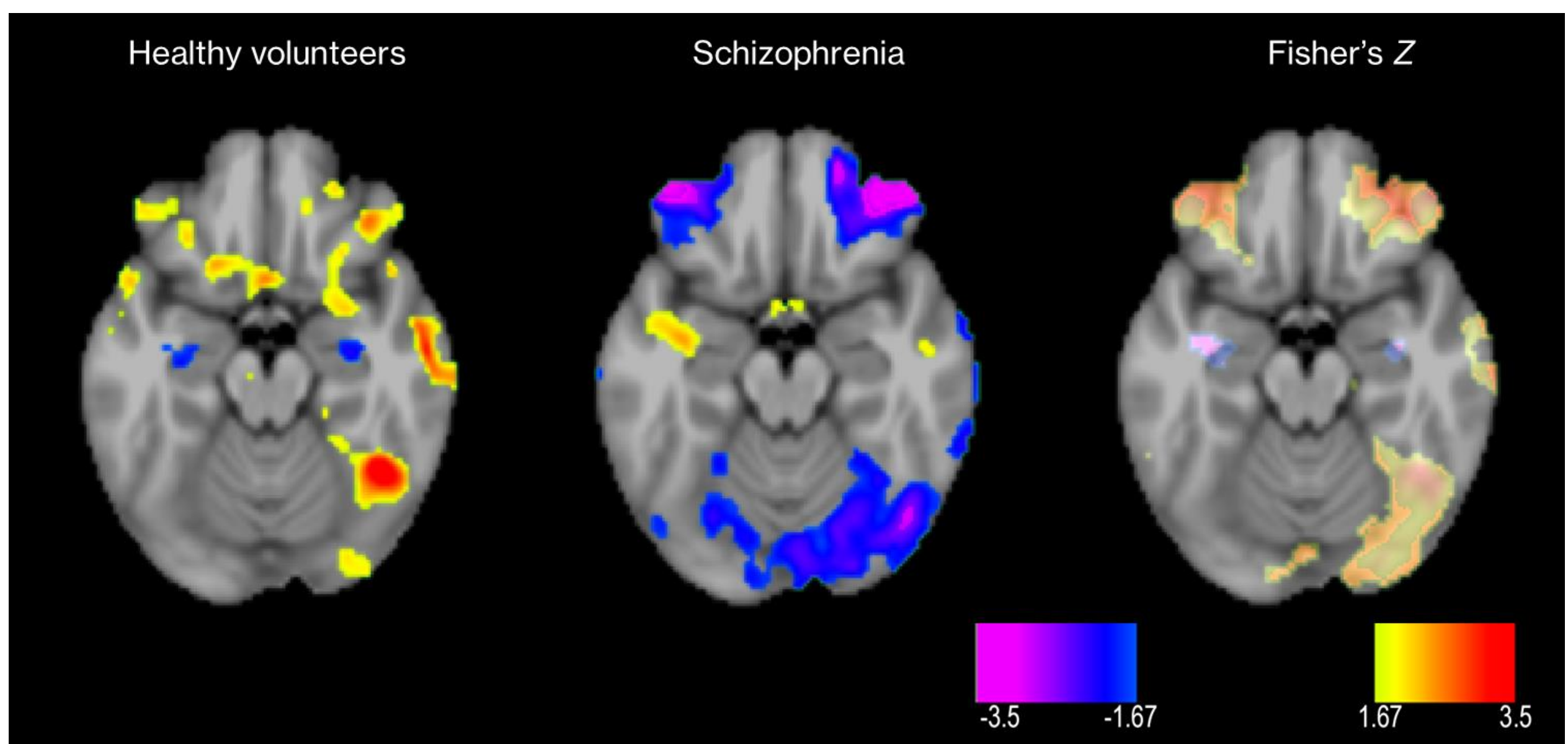

Left: Healthy volunteers' product moment correlation between learning slope and fallypride binding potential. Middle: Patients with schizophrenia Right: Fisher z-test for difference in correlations with red-yellow indicating healthy volunteers' correlations more positive than patients. Note that healthy volunteers with better performance have higher binding in frontal cortex and right temporal lobe and lower binding in parahippocampal areas. In contrast patients tended to have negative correlations between binding potential and learning slope. Color bar shows Fisher $\mathrm{Z}$ test at $\mathrm{p}<0.05$ to $\mathrm{p}<0.001,1$ tailed. 
Figure 7. Correlation differences in [F-18]fallypride binding potential between WCST categories achieved and CVLT short delay recall in healthy subjects and patients with schizophrenia.

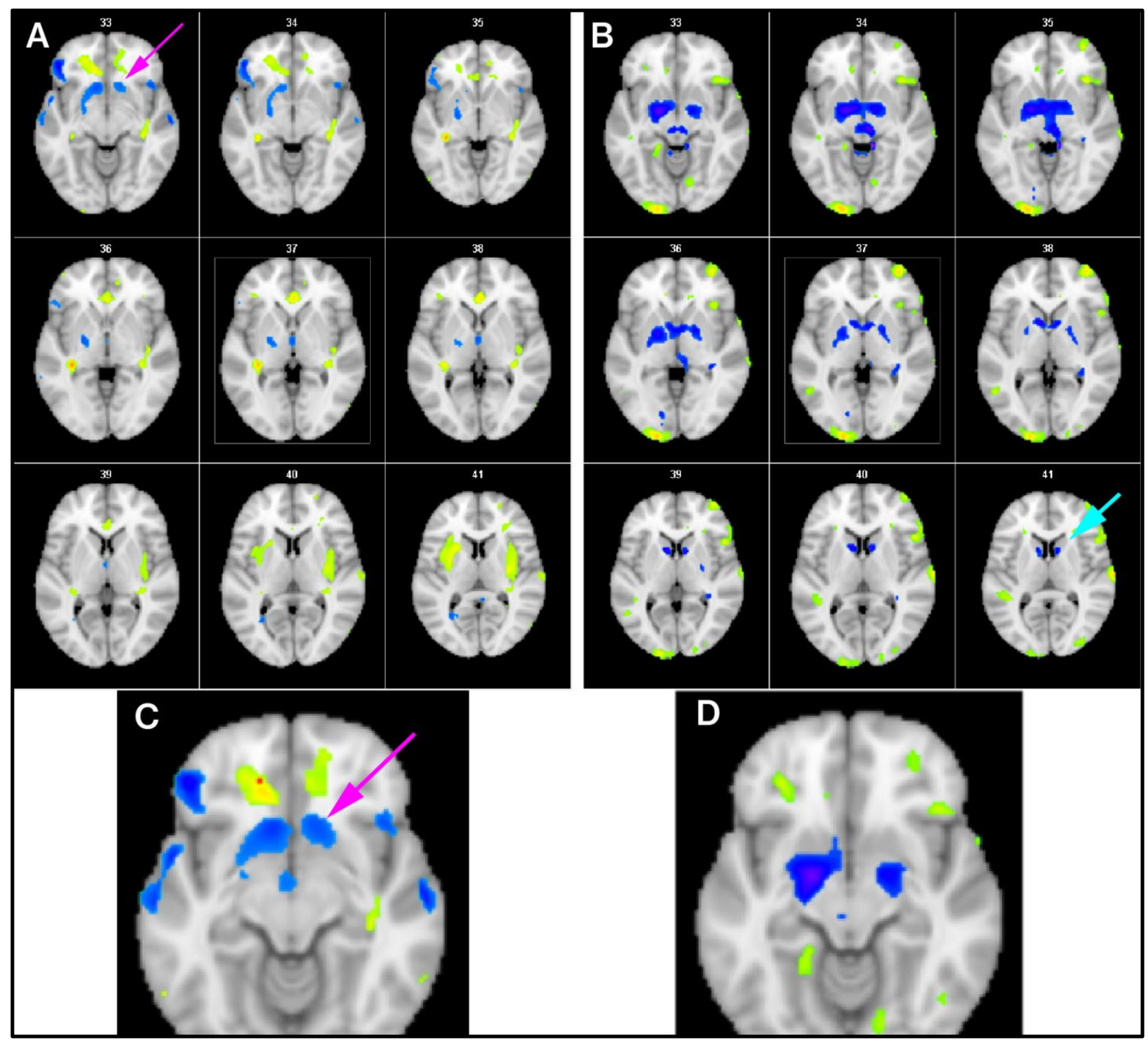

A: Fisher z-test for difference in correlations between CVLT short delay vs BP and WCST categories achieved vs. BP with blue indicating a more positive correlation with WCST. This indicates that higher binding potential is associated with better performance on short-delay recall in healthy subjects in the ventral striatum and the correlation with the WCST is lower. Thus, the $r$ correlation map of CVLT short-delay vs. binding potential (analogue of modelfree) and the $r$ correlation map of WCST categories achieved (analogue of model-based) showed a strong negative correlation in the ventral striatum (cerise arrow) but not the dorsal striatum. 
B: Patients with schizophrenia showed a different pattern with correlations missing the ventral striatum but extending into the globus pallidus ventrally and the caudate nucleus dorsally

C: Binding potential Fisher $\mathrm{Z}$ correlation difference in the ventral striatum in healthy subjects enlarged to show anatomical detail with slice between 31 and 33, upper left of panels A and B.

D: Binding potential Fisher $\mathrm{Z}$ correlation difference in the ventral thalamus region of patients with schizophrenia 